\title{
Photoacoustic imaging in the second near-infrared window: a review
}

Paul Kumar Upputuri

Manojit Pramanik 


\title{
Photoacoustic imaging in the second near-infrared window: a review
}

\author{
Paul Kumar Upputuri and Manojit Pramanik* \\ Nanyang Technological University, School of Chemical and Biomedical Engineering, Singapore
}

\begin{abstract}
Photoacoustic (PA) imaging is an emerging medical imaging modality that combines optical excitation and ultrasound detection. Because ultrasound scatters much less than light in biological tissues, PA generates high-resolution images at centimeters depth. In recent years, wavelengths in the second near-infrared (NIR-II) window (1000 to $1700 \mathrm{~nm}$ ) have been increasingly explored due to its potential for preclinical and clinical applications. In contrast to the conventional PA imaging in the visible (400 to $700 \mathrm{~nm}$ ) and the first NIR-I (700 to $1000 \mathrm{~nm}$ ) window, PA imaging in the NIR-II window offers numerous advantages, including high spatial resolution, deeper penetration depth, reduced optical absorption, and tissue scattering. Moreover, the second window allows a fivefold higher light excitation energy density compared to the visible window for enhancing the imaging depth significantly. We highlight the importance of the second window for PA imaging and discuss the various NIR-II PA imaging systems and contrast agents with strong absorption in the NIR-II spectral region. Numerous applications of NIR-II PA imaging, including whole-body animal imaging and human imaging, are also discussed. ๑ The Authors. Published by SPIE under a Creative Commons Attribution 3.0 Unported License. Distribution or reproduction of this work in whole or in part requires full attribution of the original publication, including its DOI. [DOI: 10.1117/1.JBO.24.4.040901]
\end{abstract}

Keywords: photoacoustic imaging; second near-infrared window; deep-tissue imaging; small animal imaging; contrast agents.

Paper 180571VRR received Oct. 2, 2018; accepted for publication Mar. 18, 2019; published online Apr. 9, 2019.

\section{Introduction}

In biomedical imaging, there is a huge demand for a noninvasive imaging modality that can provide high spatiotemporal resolution, high penetration depth, and anatomical and functional contrasts at an affordable price. Imaging modalities such as magnetic resonance imaging (MRI), x-ray computed tomography $(\mathrm{CT})$, single-photon emission tomography (SPET), positron emission tomography (PET), and ultrasound (US) imaging, have been widely used for clinical and preclinical imaging. ${ }^{1-5}$ Although they provide deeper penetration, leading to wholebody imaging, they have their own drawbacks. High-resolution MRI systems are expensive and slow for dynamic imaging., ${ }^{6,7}$ The $\mathrm{x}$-ray $\mathrm{CT}$ and PET/SPECT use ionizing radiation and hence may not be suitable for frequent imaging. ${ }^{8}$ US imaging cannot measure functional (physiological) changes, including metabolism, chemical composition, and absorption. ${ }^{9}$

Optical imaging modalities, including fluorescence, multiphoton fluorescence, bioluminescence, optical projection tomography, optical coherence tomography (OCT), and diffuse optical tomography (DOT), were demonstrated for in vivo animal imaging. ${ }^{10-12}$ These methods used nonionizing radiation, unlike CT scan (x-ray), SPET, and PET (gamma rays), and can provide structural as well as functional information at high resolution. ${ }^{13-16}$ However, due to the strong optical absorption and scattering of tissue, the imaging depth was limited to $\sim 0.1 \mathrm{~mm}$ (roughly the mean free path) in conventional widefield optical microscopy. ${ }^{17,18}$ OCT, on the other hand, used ballistic and quasi-ballistic photons for imaging and can image up to the transport mean free path ( $\sim 1 \mathrm{~mm}$ in biological tissue). It is currently in use for skin and eye imaging applications with an imaging depth of up to $2 \mathrm{~mm} \cdot{ }^{19,20}$ DOT offered several

*Address all correspondence to Manojit Pramanik, E-mail: manojit@ntu.edu.sg centimeters imaging depth by using diffused photons, but their image resolution was poor (approximately one-third of the depth) due to the multiple scattering the photons undergo. ${ }^{21,22}$ Recently DOT was used for monitoring neoadjuvant chemotherapy in patients with breast cancer. ${ }^{23}$ Therefore, using pure optical imaging and achieving high spatial resolution at centimeters depth was a challenging task, until photoacoustic imaging (PAI) was explored.

PAI is a hybrid imaging modality rapidly moving from bench to bedside. ${ }^{17,24-27}$ In PAI, a nanosecond-pulsed laser irradiates the tissue. On absorption of the pulsed light by the biomolecules (melanin, collagen, lipid, hemoglobin, myoglobin, bilirubin, cytochromes, water, DNA, RNA, etc.), it undergoes a time-varying thermal expansion-relaxation process, which leads to the generation of acoustic waves (known as PA waves) in the tissue. By acquiring the generated PA waves at the tissue surface and using various reconstruction algorithms, ${ }^{28-35}$ the absorption maps within the tissue can be obtained. By combining optical contrast and US resolution, PAI offers several advantages: (i) it is noninvasive and uses nonionizing radiation, hence it can be repeatedly used in vivo by keeping the excitation energy below the safety limit; (ii) it provides label-free imaging; (iii) it is speckle-free; (iv) it has higher penetration depth, up to $\sim 4 \mathrm{~cm}$ in vivo ${ }^{36}$ and $\sim 12 \mathrm{~cm}$ in vitro ${ }^{37}$ (v) it is faster and less expensive compared to MRI, PET, x-ray CT; ${ }^{38}$ (vi) it provides multiscale imaging, allows imaging organelles to organs with consistent contrast while keeping the same depth-toresolution ratio $;^{38,39}$ (vii) it provides direct imaging of optical absorption with $100 \%$ relative sensitivity-the sensitivity of PAI is 100 times higher than that of OCT and confocal microscopy; and (viii) it provides anatomical, functional, molecular, and kinetic information. ${ }^{40}$ PAI was successfully implemented as photoacoustic nanoscopy, ${ }^{41-46}$ photoacoustic microscopy (PAM) ${ }^{47-52}$ photoacoustic endoscopy, ${ }^{53-56}$ preclinical photoacoustic computed tomography/photoacoustic tomography 
(PACT/PAT) ${ }^{57-62}$ and clinical PACT. ${ }^{63-68}$ For deep-tissue imaging, PACT/PAT systems are suitable and hence hold better chance to find preclinical/clinical applications.

In the early days of PAT, green light $(\sim 532 \mathrm{~nm})$ was used due to the strong absorption of blood (an intrinsic contrast agent) in the visible region. ${ }^{69}$ Label-free in vivo PAI of cell nuclei was performed in ultraviolet region (200 to $400 \mathrm{~nm}) .^{70}$ Two major components of cell nuclei, DNA and RNA, strongly absorb UV light at wavelengths around $260 \mathrm{~nm} .^{71}$ However, due to the overwhelming scattering effect of tissues at shorter wavelengths (200 to $700 \mathrm{~nm}$ ), the intensity of light and hence the PA amplitude exponentially decreases with depth with a decay constant of a few millimeters. ${ }^{72}$ Later, PAI was performed in the near-infrared (NIR) region from 700 to $2500 \mathrm{~nm},{ }^{73}$ where the tissue attenuation is low. The first NIR window (NIR-I) from 700 to $1000 \mathrm{~nm}$ was extensively investigated for deep-tissue imaging. ${ }^{74-77}$ Owing to the reduced blood absorption in this region, the penetration depth was improved with a small sacrifice in contrast. Several contrast agents based on metallic, inorganic (carbon nanotubes, quantum dots), organic small molecules (Indocyanine green-ICG, IRDye800, methylene blue), and semiconducting nanoparticles were developed for the NIR-I window, not only to enhance the contrast but also for targeted molecular imaging, drug delivery, therapy, etc. ${ }^{78-88}$ Along with nanoparticles, reporter genes (RGs) were also used as PA agents in the range $400-800 \mathrm{~nm} .{ }^{89}$ To the best of our knowledge, RGs were not demonstrated for PAI beyond $1000 \mathrm{~nm}$. There were few drawbacks associated with PAI in NIR-I window. To produce laser light in the NIR-I range, a pump laser (typically Nd:YAG) and a second stage, Ti:Shappire, dye laser, or OPO was required. This had made the laser system expensive, bulky, and very inefficient (20\% to $30 \%$ efficiency is the best we can achieve in terms of the final laser output). Recently, pulsed laser diodes ${ }^{57,90-94}$ and light-emitting diodes ${ }^{95-100}$ were reported as alternative sources for PAT in the NIR-I window; however, they provide low-energy pulses compared to traditional lasers (few $\mathrm{mJ}$ per pulse versus hundreds of $\mathrm{mJ}$ ) and hence were not ideal for deep-tissue imaging. Another drawback of NIR-I window imaging was the low maximum permissible exposure (MPE), which was directly related to the penetration depth. For example, MPE at $800 \mathrm{~nm}$ is $\sim 300 \mathrm{~mW} / \mathrm{cm}^{2}$ and MPE at $1064 \mathrm{~nm}$ is $\sim 1000 \mathrm{~mW} / \mathrm{cm}^{2}$ per pulse according to American National Standards Institute (ANSI) safety limit. ${ }^{101}$

Recent studies on optical imaging have found a new biological window, termed as the second NIR window (NIR-II) from 1000 to $1700 \mathrm{~nm} .^{102-108}$ Second window has numerous merits, including (i) more MPE, i.e., more energy can be deposited on the tissue for imaging; this results in deeper light transmission inside tissue with longer wavelength excitation, (ii) reduced tissue scattering, thus making the tissues more transparent at longer wavelengths, (iii) minimal autofluorescence, reduces the background signal in fluorescence imaging, and (iv) maximized oxyhemoglobin absorption around $1064 \mathrm{~nm}$, thus allowing the measurement of tissue oxygenation levels deep inside the tissue. ${ }^{109}$ Unfortunately, water (one of the major endogenous chromophore) exhibits strong absorption in the range from 1400 to $1500 \mathrm{~nm}^{106}$ and hence this range of wavelengths may not be ideal for optical as well as photoacoustic deep-tissue imaging. Hence, the shorter wavelengths within the NIR-II window (1000 to $1400 \mathrm{~nm}$ ) are explored for PAI applications. ${ }^{10-115}$ The PA signal generated by NIR-II wavelengths is weak and hence effective contrast agents are often necessary for enhancing the image contrast. With the increasing demonstrations on the advantages of PAI, efforts are made to develop strong NIR-II absorbing organic, ${ }^{37,116-118}$ inorganic, ${ }^{119-126}$ etc. Although, these nanoparticles show broad absorption spectrum in the NIR-II window, most of them are demonstrated for PAI at 1064-nm wavelength because 1064-nm nanosecond laser (Nd:YAG laser) is commercially available.

In this review, we focus on the NIR-II PAI systems, contrast agents, and their preclinical and clinical applications. The review is organized in the following order: Sec. 2 discusses the motivation for NIR-II PAI, Sec. 3 discusses about in vitro imaging, Sec. 4 discusses about in vivo small animal imaging, Sec. 5 is dedicated to in vivo human imaging, Sec. 6 discusses about high-resolution imaging in the second window, Sec. 7 presents the summary and perspectives, and in Sec. 8, we conclude the review.

\section{Motivation for Near-Infrared-II Photoacoustic Imaging}

Typical processes that are involved in light-tissue interactions when a laser beam penetrates through biological tissues are reflection, autofluorescence, scattering, and absorption. ${ }^{127}$ In photoacoustics, the imaging depth is majorly governed by absorption and scattering coefficients of the tissue. The absorption coefficients are strongly affected by the presence of blood, particularly at wavelengths below $600 \mathrm{~nm}$ (in the visible region). Figure 1(a) shows the absorption coefficients of tissue chromophores, including oxyhemoglobin $\left(\mathrm{HbO}_{2}\right)$, deoxyhemoglobin $(\mathrm{Hb})$, lipid, and water in the range from 200 to $1400 \mathrm{~nm}$. From this graph, it is clear that in the NIR region the blood absorption is low, and hence the penetration depth is majorly decided by the scattering of light and absorption of water in biological tissues. A study on human brain tissue found that the region from 1000 to $1100 \mathrm{~nm}$ can provide better penetration depths for brain tissues. The results have proven that in the 1000- to 1100-nm region the scattering coefficients reduce with wavelength, absorption coefficient is quite low, and water absorption is still weak. This study has been supported by Monte Carlo simulations of light transportation in brain tissue. $^{129}$ The optical absorption and scattering coefficients of human breast tissues are identified for wavelengths from 500 to $1100 \mathrm{~nm}$. It is found that the absorption coefficients are high at below $600 \mathrm{~nm}$ due to blood. The scattering coefficients lie in the range from 30 to $90 \mathrm{~mm}^{-1}$ at $500 \mathrm{~nm}$ and fall smoothly with increasing wavelength between 10 and $50 \mathrm{~mm}^{-1}$ at $1100 \mathrm{~nm} .{ }^{130}$ For skin and other tissues, the scattering coefficient decreases with an increase in wavelength, as shown in Fig. 1(b). ${ }^{127,131-135}$ All these simulations and experimental studies have proved that wavelengths in the NIR-II region are more suitable for biomedical imaging.

For in vivo PAI, the maximum permissible pulse energy and the maximum permissible pulse repetition rate are governed by the ANSI laser safety standards. ${ }^{101}$ The safety limits for the skin depend on the optical wavelength, pulse duration, exposure duration, and exposure aperture. For exposure time 10 to $3 \times 10^{4} \mathrm{~s}$ and spectral range from 0.4 to $1.4 \mu \mathrm{m}$, according to ANSI safety limit, the MPE $=0.2 C_{A} \mathrm{~W} / \mathrm{cm}^{2}$, where, $C_{A}=1.0$ (for 0.4 to $0.7 \mu \mathrm{m}$ ); $10^{2(\lambda-0.7)}$ (for 0.7 to $1.05 \mu \mathrm{m}$ ); and 5.0 (for 1.05 to $1.4 \mu \mathrm{m}$ ). Similarly, for the exposure time 10 to $3 \times 10^{4} \mathrm{~s}$ and spectral range from 1.4 to $1.8 \mu \mathrm{m}$, the MPE $=$ $0.1 \mathrm{~W} / \mathrm{cm}^{2}$. For the spectral range from 0.4 to $1.8 \mu \mathrm{m}, \mathrm{MPE}$ as a function of wavelength is shown in Fig. 1(c). This graph 

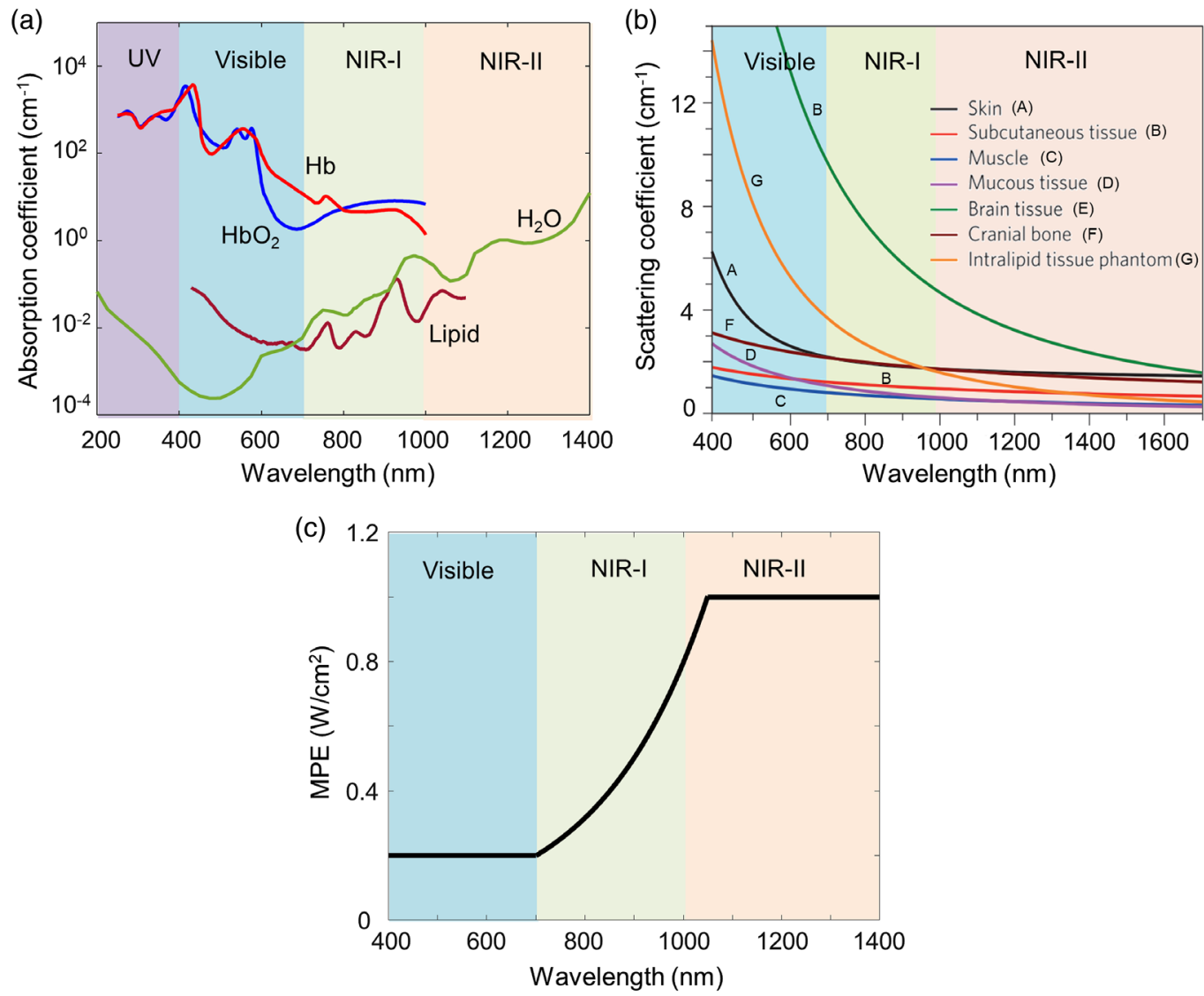

Fig. 1 (a) Absorption coefficient spectra of endogenous tissue chromophores at their typical concentrations in the human body. Adapted from Ref. 128. (b) Reduced scattering coefficients of different biological tissues and of Intralipid scattering tissue phantom as a function of wavelength in the 400- to $1700-\mathrm{nm}$ region, which covers the visible, NIR-I, and NIR-Il windows (blue, green, and red shaded regions, respectively). They all show reduced scattering at longer wavelengths. Reproduced with permission from Ref. 127. (c) MPE as a function of excitation wavelength.

shows that the NIR-II window allows five times higher MPE for deep-tissue imaging compared to visible windows.

Implementing NIR-II PAI is comparatively straightforward. In conventional PAT systems, the excitation source is a pump laser (typically Q-switched 1064/532 nm Nd:YAG laser) and a second-stage OPO tunable in the range from 670 to $970 \mathrm{~nm}$. For NIR-I PAI, 670 to $970 \mathrm{~nm}$ is used. OPO laser typically have $12 \%$ to $15 \%$ efficiency of generating light in the NIR-I range. For example: if a Nd:YAG pump laser produces $8 \mathrm{~W}$ of laser energy at $1064 \mathrm{~nm}$, when it is converted to $532 \mathrm{~nm}$, we get roughly $50 \%$ to $60 \%$ efficiency, i.e., $\sim 4-\mathrm{W}$ energy. Further, when the 532-nm light is converted to NIR-I laser, we get around 100- to 130-mW energy. For NIR-II imaging, the powerful laser output from $1064 \mathrm{~nm} \mathrm{Nd:YAG} \mathrm{laser} \mathrm{can} \mathrm{be} \mathrm{used.} \mathrm{The}$ idler wavelengths of OPO tunable in the range from 1200 to $2000 \mathrm{~nm}$ can also be used for NIR-II imaging, but they have very low energy. Thus, the availability of high-power 1064-nm Nd:YAG laser has helped researchers to quickly start investigation on NIR-II window. However, a high-power laser tunable in the NIR-II window is still needed to fully utilize the benefits offered by this window. Thus, the reduced blood absorption, reduced scattering coefficients, high MPE offered by NIR-II window, and availability of high-power 1064-nm Nd:YAG nanosecond laser have motivated researchers to investigate its usefulness in PAI.

\section{In Vitro Imaging}

Biological tissues are generally opaque because photons traveling in the tissues are predominantly scattered rather than absorbed. Deep-tissue imaging becomes necessary to examine blood vessels, different organs (brain, kidney, liver, heart, etc.), and lesions/ tumors for preclinical/clinical applications. Scattering, absorption, and hence the imaging depth are strongly influenced by the excitation wavelength. Owing to the reduced scattering at NIR-II wavelengths, longer penetrations can be achieved. In this section, we discuss the deep-tissue imaging applications of PAT system operated at $1064 \mathrm{~nm}$.

\subsection{Deep-Tissue Imaging}

PAT offers high depth-to-resolution ratio compared to pure optical imaging methods. An imaging depth of $\sim 5 \mathrm{~cm}$ is achieved in chicken breast tissue at $1064 \mathrm{~nm} .{ }^{136}$ A semiconducting polymer [named as poly (thienoisoindigo-alt-diketopyrrolopyrrole)PIGD] is the contrast agent. Figure 2(a) shows the schematic representation of the PIGD nanoparticle. These particles are spherical, with an average diameter of $21 \pm 5 \mathrm{~nm}$. This dispersion results in a broad spectrum covering the range from 800 to $1200 \mathrm{~nm}$ with a maximum at $960 \mathrm{~nm}$, as shown in Fig. 2(b). The homemade PAT system used for deep-tissue imaging is shown in Fig. 2(c). The excitation source is Nd:YAG laser (1064 nm, $5 \mathrm{ns,}$ 

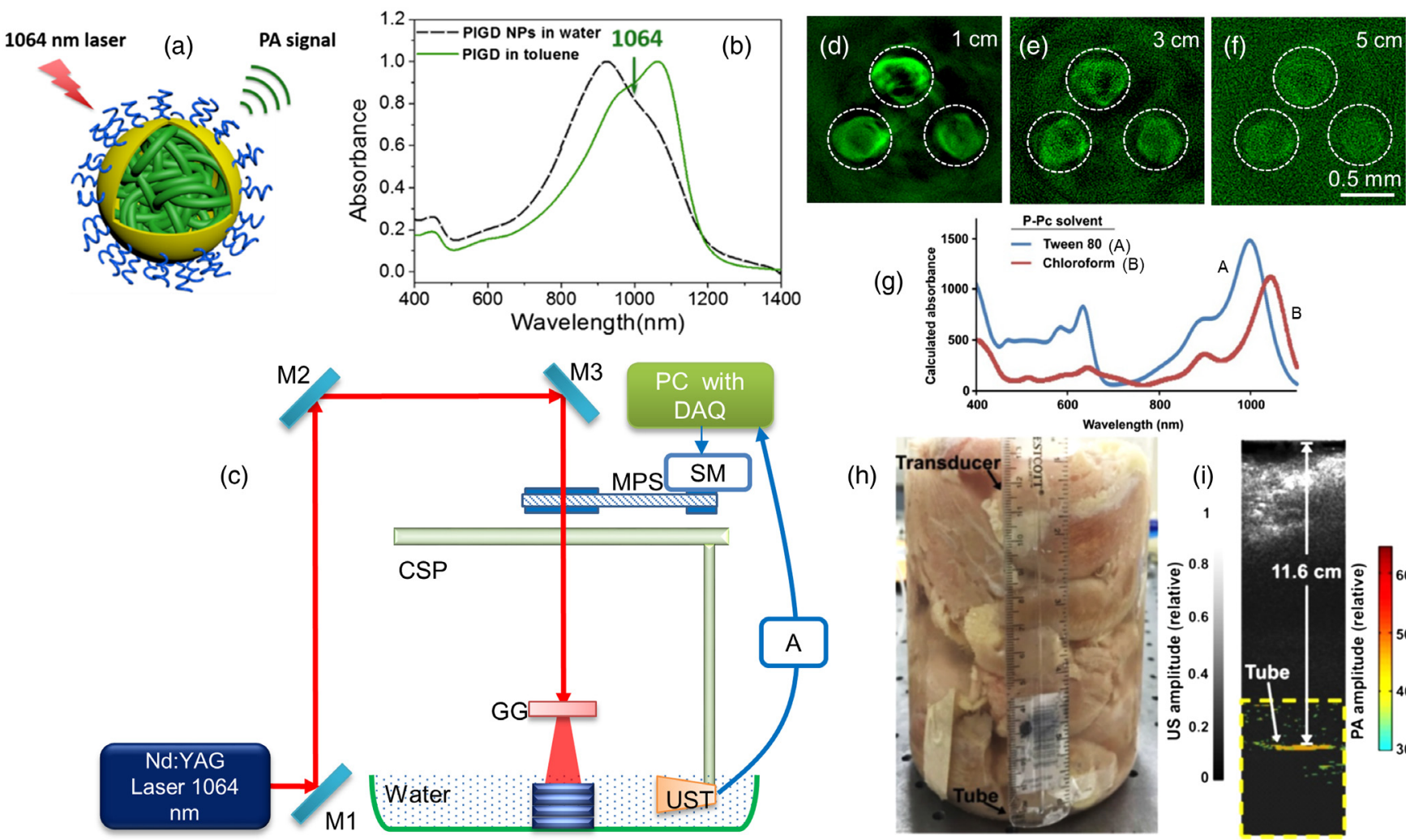

Fig. 2 (a) Schematic illustration of the conjugated PIGD encapsulated by surfactant F127 upon nanoprecipitation in water/Tetrahydrofuran (THF) mixture followed by evaporation of THF, resulting in colloidal nanoparticles with PIGD as the core, PPO as the shell, and PEO as the corona. (b) UV-vis-NIR absorption spectra of PIGD in water/toluene; (c) schematic representation of 1064-nm PAT system that is used for imaging PIGD NPs embedded inside the chicken breast tissue at $1064 \mathrm{~nm}$. (d)-(f) PA images of the agar gel phantom containing PIGD NPs solutions with different concentrations ( $\mathrm{s} 1-\mathrm{s} 3: 1.0,0.5,0.25 \mathrm{mg} /$ $\mathrm{mL}$, respectively) acquired at different depths, and laser energy density used on tissue surface is $\sim 25 \mathrm{~mJ} / \mathrm{cm}^{2}$. Reproduced with permission from Ref. 136. Detection of P-Pc through $11.6 \mathrm{~cm}$ of tissue: (g) optical absorption spectrum of P-Pc, (h) photograph of the sample setup with chicken breast stacked on top of a tube containing the P-Pc formulation, (i) overlaid photoacoustic (color, within the dashed box) and US (gray) images of the tube at 11.6-cm depth. Reproduced with permission from Ref. 37.

$10 \mathrm{~Hz}$ ) from Continuum, Surelite Ex. The 1064-nm beam is expanded and homogenized using an optical ground glass to illuminate the sample. The sample and the ultrasonic transducer (UST) are immersed in water for coupling the PA signal to the transducer. A nonfocused transducer (V323-SU/2.25 MHz, 0.5 inch, Olympus NDT) is used for collecting the signals. The signals are subsequently amplified, bandpass-filtered by an US pulser/receiver unit (AU), (Olympus NDT, 5072PR) and then digitized and recorded by the PC with a data acquisition (DAQ) card (25 Ms/s, GaGe, compuscope 4227). For the cross-sectional imaging, the transducer is driven by a computer-controlled stepper motor to continuously move in a circular motion. The acquired A-lines are used for reconstructing the image using a delay-and-sum backprojection algorithm. The solutions of PIGD Nanoparticles (NPs) with three different concentrations $(1.0,0.5$, and $0.25 \mathrm{mg} / \mathrm{mL})$ are embedded in an agar gel phantom. The phantom is placed under chicken breast tissues with different thicknesses. Images of the phantom at different depths are shown in Figs. 2(d)-2(f). All three spots are detectable at the tissue depth of up to $5 \mathrm{~cm}$ [Fig. 2(f)]. The 1064-nm energy used on the tissue surface is $\sim 25 \mathrm{~mJ} / \mathrm{cm}^{2}$ (less than the ANSI limit of $\sim 100 \mathrm{~mJ} / \mathrm{cm}^{2}$ ). The $5-\mathrm{cm}$ imaging depth promises that PIGD NPs could act as a potential agent for noninvasive deep-tissue imaging.
A linear-array-based PAT demonstrated an in vitro $\sim 12-\mathrm{cm}$ imaging in chicken tissue using phosphorus phthalocyanine (P-Pc) as contrast agent. ${ }^{37}$ P-Pc showed strong absorption around $1000 \mathrm{~nm}$, as shown in Fig. 2(g). The imaging system includes $1064 \mathrm{~nm} \mathrm{Nd:YAG} \mathrm{excitation} \mathrm{laser} \mathrm{and} \mathrm{a} \mathrm{128-element}$ linear transducer array (5 MHz, ATL/Philips L7-4) for collecting PA signals. A Tygon tube (35-mm length, 5-mm inner diameter) containing $30 \mathrm{mM}$ of P-Pc was placed in a 2-L glass beaker whose bottom was covered with 2.5 -cm-thick chicken breast tissue [Fig. 2(h)]. To prevent leakage, both ends of the tube were sealed with epoxy glue. Figure 2(i) shows the PA/US image obtained after averaging 100 images acquired at the same depth of $11.6 \mathrm{~cm}$. The image signal-to-noise ratio (SNR) was $18 \mathrm{~dB}$ and the energy on the tissue was $\sim 56 \mathrm{~mJ} / \mathrm{cm}^{2}$ (still less than the ANSI safety limit). The deeper penetration was mainly attributed to the high absorption of P-Pc, weak tissue absorption and scattering, and the higher ANSI safety limit at $1064 \mathrm{~nm}$. The 11.6-cm imaging depth achieved with $30 \mathrm{mM}$ $\mathrm{P}-\mathrm{Pc}$ at $1064 \mathrm{~nm}$ was significantly higher than the 8.4-cm depth achieved with $30 \mathrm{mM}$ methylene blue at $650 \mathrm{~nm} .{ }^{137}$ Although these experiments were performed in chicken tissues, the deep detection of NIR-II nanoparticles through 5- to 12-cm-thick biological tissue showed its potential for preclinical applications that require whole-body penetration in small animals. 


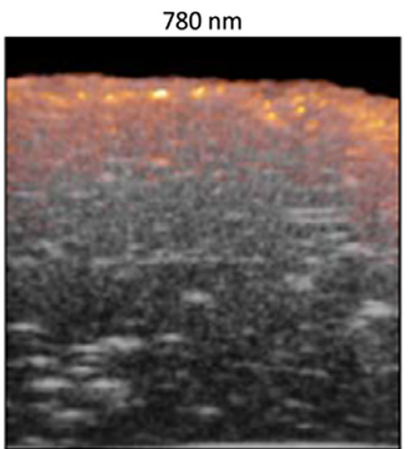

(a)

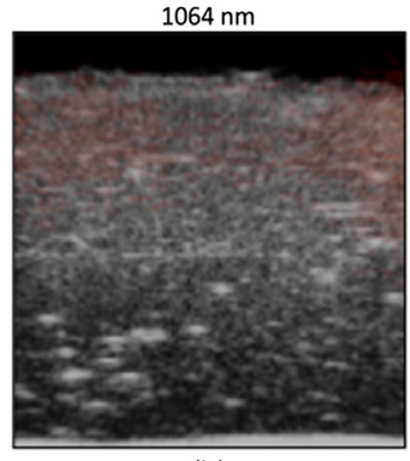

(b)

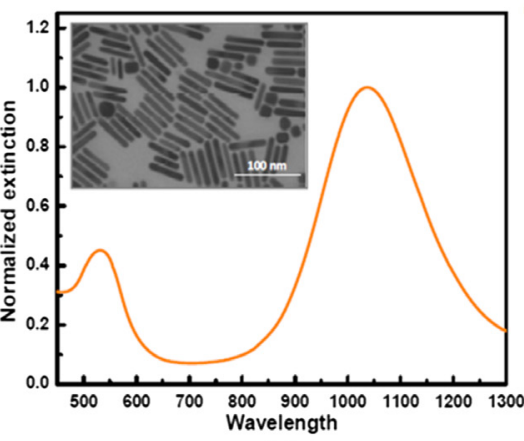

(c)

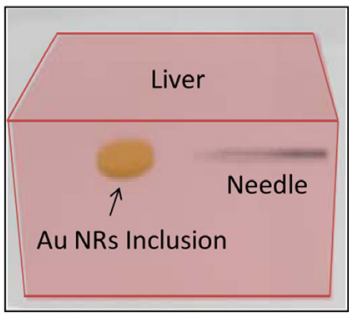

(d)

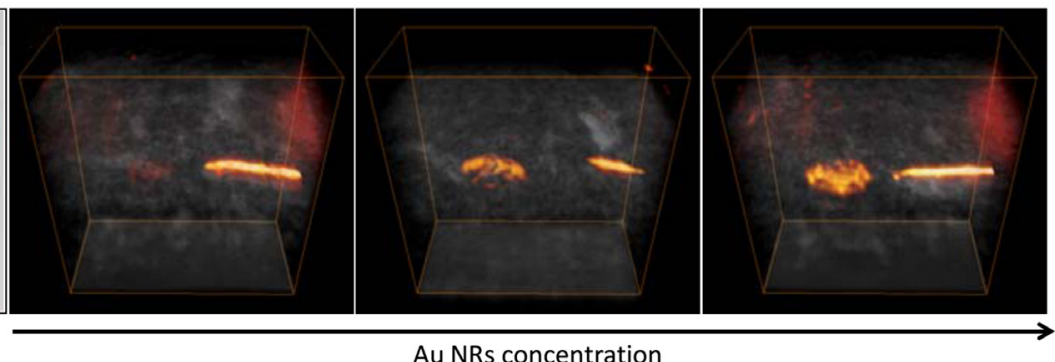

(e)

Fig. 3 Combined US (gray) and photoacoustic (red) images of an ex vivo bovine liver at wavelengths of (a) $780 \mathrm{~nm}$ and (b) $1064 \mathrm{~nm}$. (c) Optical spectrum of gold (Au) nanorods (inset shows a TEM image of the Au nanorod, the scale bar is $100 \mathrm{~nm}$ ). (d) Schematic illustration of the liver phantom with Au nanorod inclusions and one embedded needle. (e) Combined PA/US images of the liver phantom captured at $1064 \mathrm{~nm}$ with increasing concentrations of Au nanorods in the inclusion from left to right $(5,12.5$, and $25 \mu \mathrm{g} / \mathrm{mL}$, respectively). Reproduced with permission from Ref. 124.

\subsection{Liver Imaging}

Liver is the largest internal organ in the human body. After lymph nodes, liver is the second most common site of metastasis for cancers, such as pancreas, breast, colon, and stomach. Therefore, it is important to screen the formation of metastatic lesions in patients with cancer history. US, x-ray CT, PET, and MRI have been used for imaging liver lesions. Recently, the feasibility of PAT for imaging liver lesions ex vivo has been demonstrated at $1064 \mathrm{~nm}$ using gold nanorods. ${ }^{124}$ The PAI contrast mostly depends on optical absorption of blood as compared to the background tissue. It is challenging to image liver lesions with PAT, because at any given time liver contains $\sim 10 \%$ of total blood in the body and it filters $\sim 1.4 \mathrm{~L}$ of blood per minute in adults. Owing to the strong absorption of blood, imaging liver is easy but imaging liver lesion/tumor with good contrast is inherently difficult for PAT operated in visible or NIR-I region. Liver phantom images are compared at $780 \mathrm{~nm}$ (NIR-I) and $1064 \mathrm{~nm}$ (NIR-II) in Figs. 3(a) and 3(b), respectively. The liver tissue generates a strong PA signal at $780 \mathrm{~nm}$ (due to the strong absorption of hemoglobin) compared to $1064 \mathrm{~nm}$. By using 1064-nm excitation and strong 1064-nm absorbing agent, liver lesions imaging with high contrast is possible.

Gold (Au) nanorods resonant at $1064 \mathrm{~nm}$ are used as contrast agent for liver imaging. The absorption spectrum of PEGylated $\mathrm{Au}$ nanorods is shown in Fig. 3(c); inset shows transmission electron microscopic (TEM) image of the nanorods. Figure 3(d) shows the schematic illustration of the liver phantom. Figure 3(e) shows a series of PA/US images obtained at $1064 \mathrm{~nm}$. The Au nanorod concentrations used in the inclusion are 5, 12.5, and $25 \mu \mathrm{g} / \mathrm{mL}$, respectively. An increased PA response from these inclusions has proved that plasmonic gold nanorods absorbing at $1064 \mathrm{~nm}$ can serve as a PA agent for liver imaging. The contrast of the images can be attributed to a combined effect from the suppression of background signal (due to the reduced blood absorption at $1064 \mathrm{~nm}$ ) and the strong absorption of 1064-nm wavelength by the plasmatic gold nanorods. Although the in vitro studies are promising, lot more work is needed to study the feasibility of this approach for in vivo animals before going into clinical trials.

\subsection{Brachytherapy}

Brachytherapy is a form of radiation therapy commonly used in the treatment of prostate cancer. It involves implantation of small radioactive seeds around the treatment area. Currently, US imaging is used for seed implantation, but the seeds are difficult to distinguish from the tissue structure due to artifacts. Combined photoacoustic + US system operated at $1064 \mathrm{~nm}$ (NIR-II) is demonstrated for identifying brachytherapy seeds (BTSs) implanted at 5-cm deep inside the tissue phantom. ${ }^{138}$ The PA + US system and schematic representation of the tissue phantom are shown in Figs. 4(a) and 4(b), respectively. The system includes a research US system (VDAS-I, Verasonics) and a 1064-nm Surelite III/OPO Plus (Continuum) providing tunable light at 650 to $900 \mathrm{~nm}$. A 128-element L7-4 (5 MHz) linear array transducer (AT5L40B, Broadsound Corp.) is used to acquire acoustic signals. The gray color tube is titanium-shelled 4.5-mm-long by $0.8-\mathrm{mm}$ diameter BTSs (IAI-125A nonradioactive seeds, IsoAid LLC, Port Richey). To compare the contrast of BTSs with the endogenous contrast, a tube having an inner diameter of $\sim 0.86 \mathrm{~mm}$, and length $\sim 4 \mathrm{~cm}$ is filled with rabbit 
(a)

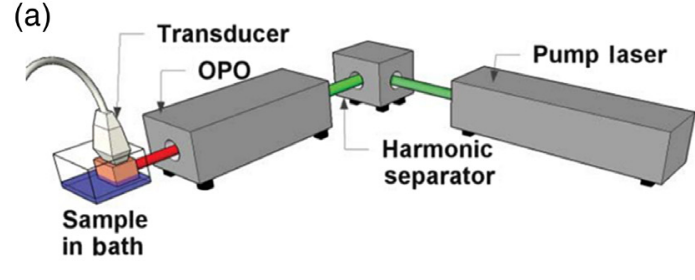

(b)

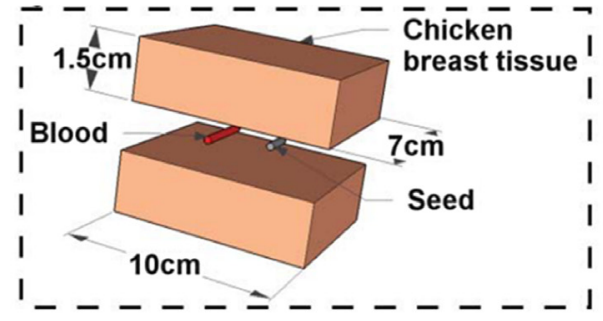

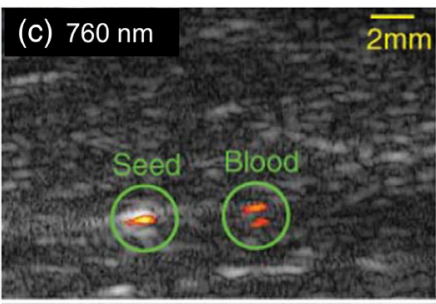
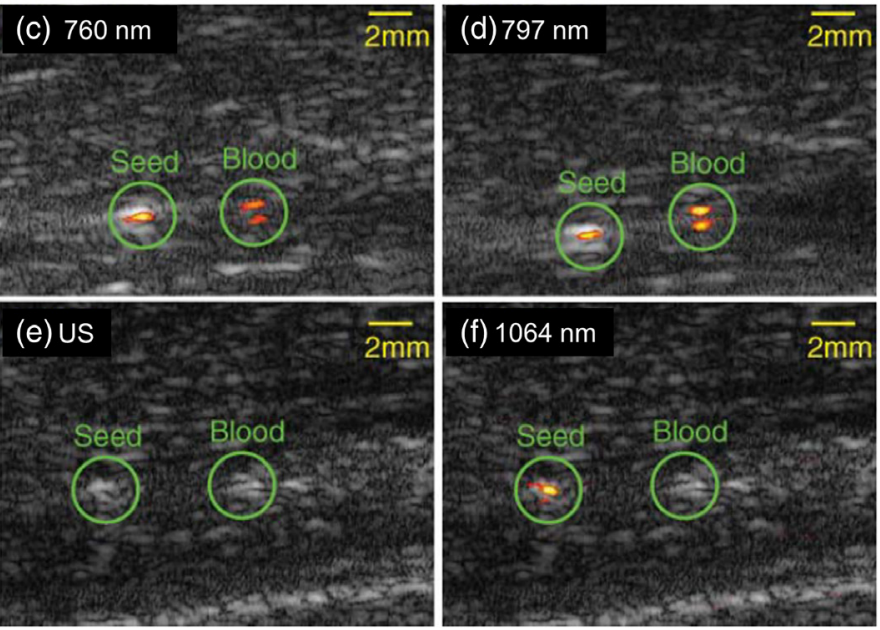

Fig. 4 (a) Schematic representation of the PA/US system. (b) Schematic representation of the tissue phantom with relevant dimensions. (c) and (d) Combined PA/US images at a laser fluence of $20 \mathrm{~mJ} / \mathrm{cm}^{2}$ and a laser penetration depth of $2 \mathrm{~cm}$. (e) and (f) US and combined PA/US images at a fluence of $100 \mathrm{~mJ} / \mathrm{cm}^{2}$ and a laser penetration depth of $5 \mathrm{~cm}$. Reproduced with permission from Ref. 138.

blood. The phantom [Fig. 4(b)] is imaged at different wavelengths and different tissue depths. The PA + US images at 760 and $797 \mathrm{~nm}$ are shown in Figs. 4(c) and 4(d), respectively. In these images, seed-to-blood contrast-to-noise ratios are 15 and $-0.5 \mathrm{~dB}$, respectively. In the US image [Fig. 4(e)], the BTSs and the blood-filled tube are indistinguishable. But the overlaid PA signal correctly identifies the seed [Fig. 4(f)]. At $1064 \mathrm{~nm}$, the blood-filled tube is indistinguishable from the background noise, whereas the BTSs are clearly visible. It should be noted that in this study the MPEs $20 \mathrm{~mJ} / \mathrm{cm}^{2}$ at 760 and $797 \mathrm{~nm}$ and $100 \mathrm{~mJ} / \mathrm{cm}^{2}$ at $1064 \mathrm{~nm}$ are used. The study showed that, by using $100 \mathrm{~mJ} / \mathrm{cm}^{2}$ at $1064 \mathrm{~nm}$, the BTSs at 5-cm-depth inside the chicken tissue are detected. It is a proof-of-concept study, but more rigorous in vivo animals and clinical studies should be undertaken.

\section{In Vivo Small Animal Imaging}

Small animals (especially rats, mice, etc.) are suitable models for preclinical studies, and they play an important role in studying human diseases and their effective treatments. ${ }^{139}$ Standard preclinical imaging methods include MRI, x-ray CT, PET, UST, and optical methods (fluorescence, DOT, etc.). As an alternative to these modalities, PAT has emerged as a preclinical tool. Initially, visible window ${ }^{140,141}$ and later NIR-I window ${ }^{141-145}$ had been used for in vivo animal imaging. In this section, we discuss the advanced PAT systems operated in NIR-II window for preclinical studies.

\subsection{Whole-Body Imaging}

Whole-body imaging of mouse was performed in visible and NIR-I windows. ${ }^{146-148}$ Recently, using hemoglobin contrast, different organs (brain, heart, liver, and kidneys) of mouse were imaged using PAT at $1064 \mathrm{~nm} .{ }^{149}$ The imaging system used a compact high-energy 1064-nm diode-pumped Nd:YAG laser [Fig. 5(a)] with the following specifications: $3.2(L) \times$ $14.0(W) \times 6.5(H) \mathrm{cm}$, weight $\sim 1.6 \mathrm{~kg}, \mathrm{M}^{2}$-factor (beam quality factor) $\sim 3$, pulse energy $\sim 80 \mathrm{~mJ}$, and pulse repetition rate 1 to $50 \mathrm{~Hz}$, from Montfort Laser GmbH, Austria. The schematic representation of the PAT design used for trunk imaging is shown in Fig. 5(b). For brain imaging, 1064-nm light with $\sim 12 \mathrm{~mJ} / \mathrm{cm}^{2}$ energy was delivered to the scalp through a $\sim 1.2$-cm diameter fiber bundle. Because the cortex is $\sim 1 \mathrm{~mm}$ beneath the scalp surface, top illumination was used. For trunk imaging, the light delivery was provided by a 6-cm-diameter ring fiber illuminator (Fiberoptic Specialties Light Source Flex Cable 12-Light RingMount). Along the ring, 12 circular fiber outputs $(0.3 \mathrm{~cm}$ in diameter) were evenly distributed and were illuminating toward the ring center at a 60-deg angle (along the elevation direction). The PA signals were detected by a 270 -deg ring transducer array (128 elements, $5 \mathrm{MHz}, 40-\mathrm{mm}$ ring diameter, 35-mm elevation focus). Brain, liver, and kidney regions were imaged with $10-\mathrm{Hz}$ pulse repetition frequency over $50 \mathrm{~s}$, whereas the heart region was imaged with $50 \mathrm{~Hz}$ pulse repetition frequency over $10 \mathrm{~s}$. The tomography of mouse brain vasculature and liver has been shown in Figs. 5(c) and 5(d), respectively. These results clearly showed the high-speed imaging capacity of the 1064-nm PAT system.

An advanced preclinical system, called the single-impulse panoramic PACT (SIP-PACT), was reported for the in vivo whole-body imaging of small animals at $1064 \mathrm{~nm} .{ }^{150}$ For whole-body imaging, a 1064-nm laser beam $(50 \mathrm{~Hz}, 5$ to $9 \mathrm{~ns}$, DLS9050, Continuum) was expanded by an engineered diffuser (EDC10-A-1r, RPC Photonics) and then was passed through a conical lens (AX-FS-1-140-0, Del Mar Photonics) to form a ring-shaped beam which was refocused by an optical condenser. The 1064-nm light with $\sim 18 \mathrm{~mJ} / \mathrm{cm}^{2}$ energy formed a ring pattern on the trunk of the mouse. For a two-dimensional (2-D) panoramic detection, a 512-element full-ring transducer (Imasonic Inc., 50-mm ring radius, $5 \mathrm{MHz},>90 \%$ one-way bandwidth) was used. Images were formed by a half-time dualspeed-of-sound universal backprojection algorithm. ${ }^{151}$ The schematic representation of the system used for trunk imaging is shown in Figs. 5(e) and 5(f). The anatomy of different organs (brain, heart, lungs, liver, spleen, kidney, caecum, intestine, etc.) of the mouse was obtained with hemoglobin contrast. Figures 5(g)-5(i) show the cross section of brain cortex, lower thoracic cavity, and upper abdominal cavity, respectively. The 

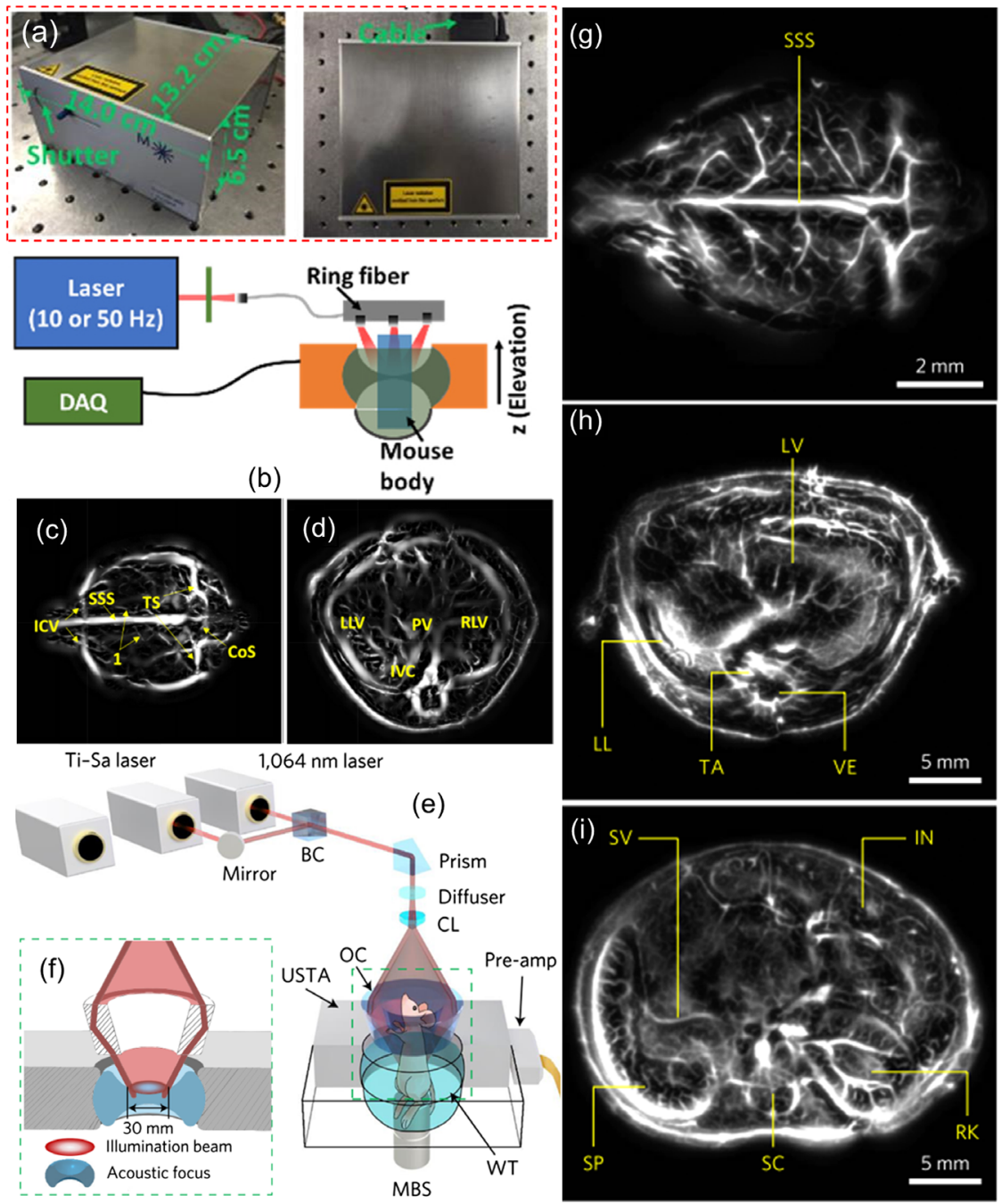

Fig. 5 (a) Photographs of the diode-pumped Nd:YAG laser. (b) Schematic representation of the setup used for in vivo trunk imaging of the mouse. (c) Cerebral vasculature of the mouse brain. (d) Image of the liver region. CoS, confluence of sinuses; SSS, superior sagittal sinus; ICV, inferior cerebral vein; TS, transverse sinus; LLV, left lobe of liver; PV, portal vein; RLV, right lobe of liver; and IVC, inferior vena cava. Reproduced with permission from Ref. 149. In vivo whole-body imaging of the mouse using SIPPACT system: (e) schematic representation of the system used for trunk imaging; (f) close up of the green dashed box region in Fig. 4(e), which shows the confocal design of light delivery and photoacoustic detection. BC, beam combiner; CL, conical lens; MBS, magnetic base scanner; OC, optical condenser; USTA, full-ring ultrasonic transducer array; WT, water tank. Label-free SIP-PACT of the mouse wholebody anatomy from the brain to the trunk: $(\mathrm{g})$ vasculature of the brain cortex, cross-sectional images of the (h) upper thoracic cavity, (i) lower thoracic cavity, (j) two lobes of the liver, (k) upper abdominal cavity, and (I) lower abdominal cavity. IN, intestines; LL, left lung; LV, liver; RK, right kidney; SC, spinal cord; SP, spleen; SV, splenic vein; TA, thoracic aorta; and VE, vertebra. Reproduced with permission from Ref. 150.

whole body of the mouse having a trunk width of $\sim 28 \mathrm{~mm}$ was clearly imaged with a spatial resolution of $125 \mu \mathrm{m}$ and an imaging frame rate of $50 \mathrm{~Hz}$. Thus, the NIR-II (1064 nm) excitation combined with advanced data acquisition and processing tools enabled SIP-PACT to complement other small-animal whole-body anatomical imaging modalities.

\subsection{Brain Imaging}

In vivo brain imaging was demonstrated in NIR-I and NIR-II windows using semiconducting polymer nanoparticles (SPNs). The details of the SPN-I having absorption in NIR-I, and SNP-II having absorption from visible to NIR-II regions can be found in
Ref. 152. The schematic representation of SPN-II nanoparticle and optical absorption spectra of SPN-I and SPN-II are shown in Figs. 6(a) and 6(b), respectively. The SPN-II showed a spectrum ranging from visible to NIR-II region with the maximum peak at $1253 \mathrm{~nm}$. The SPN-II $(6 \mathrm{mg} / \mathrm{mL}, 300 \mu \mathrm{L}$ per rat) was injected into the rat through intravenous injection. After injection, brain images were acquired using the homemade PAT system, as shown in Fig. 6(c). Postinjection brain images were acquired at $750 \mathrm{~nm}$ for up to $70 \mathrm{~min}$. It was observed that the PA signals from the blood vessels increased by $66 \%$ and the SNR was enhanced by about 1.5 -fold after injection. The SNR was stabilized for $70 \mathrm{~min}$, indicating the long circulation of SPN-II in blood owing to its small diameter $(\sim 54 \mathrm{~nm})$ and PEG-passivated 


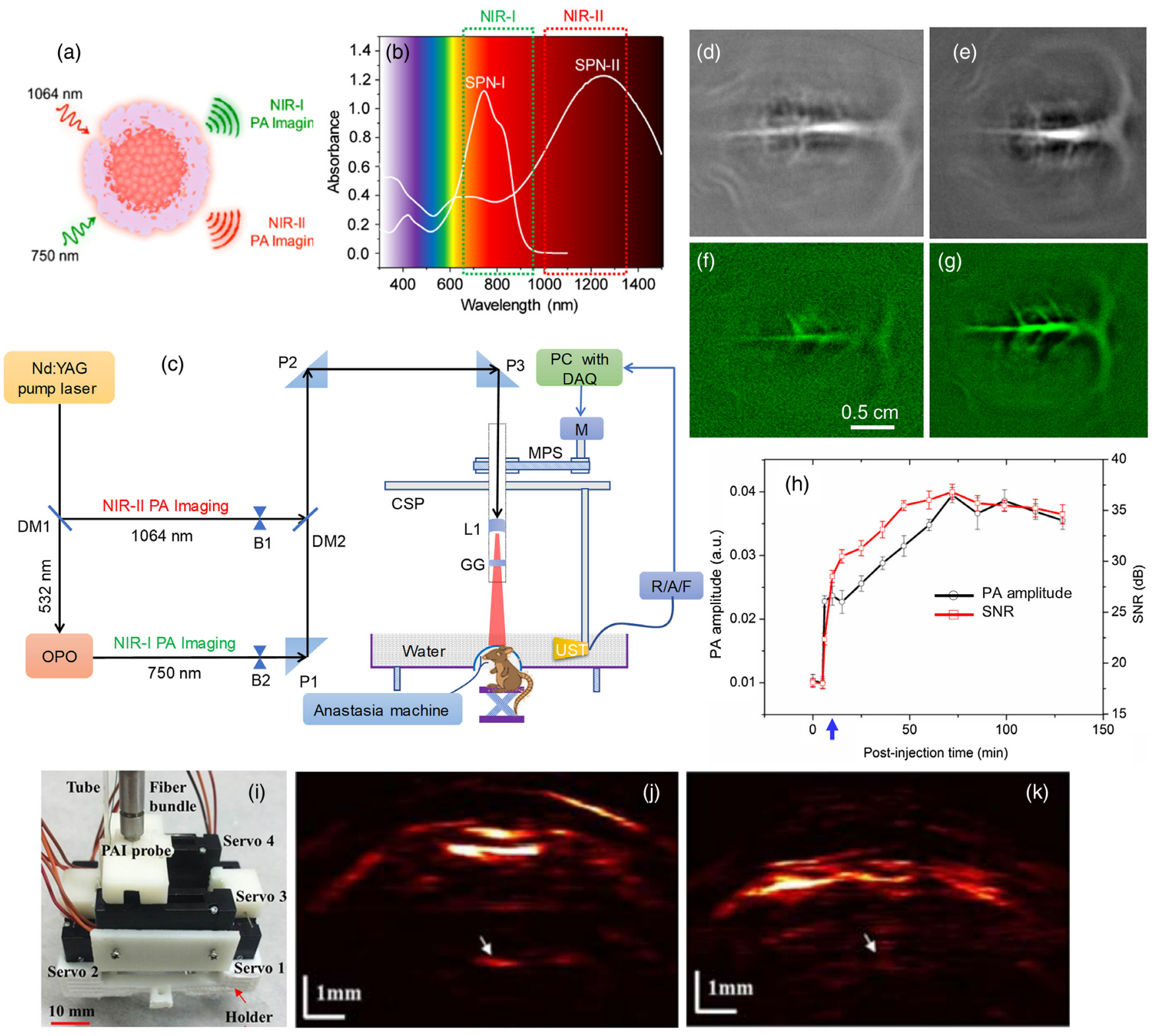

Fig. 6 (a) Schematic representation of the SPN-II nanoparticles. (b) Optical absorption spectra of SPN-I and SPN-II $(40 \mu \mathrm{g} / \mathrm{mL})$. (c) Schematic representation of the PAT system for both NIR-I imaging and NIRIl imaging: DM, dichromic mirror; B, beam blocker; $\mathrm{P}$, antireflection-coated right-angle prism; MPS, motor pulley system; CSP, circular scanning plate; L1, concave lens; GG, ground glass; DAQ, data acquisition card; M, stepper motor; R/A/F, US signal receiver, amplifier, and filter; and UST, ultrasonic transducer. In vivo PA images of the rat cortex at $750 \mathrm{~nm}$ (d) and $1064 \mathrm{~nm}(\mathrm{e})$ that are acquired 70 min after injecting SPN-II (6 mg/mL, $0.3 \mathrm{~mL}$ per rat). Laser energy density has been set at $\sim 5.5 \mathrm{~mJ} / \mathrm{cm}^{2}$. Reproduced with permission from Ref. 152. In vivo PAl of rat brain vasculature: cross-sectional brain vascular images at (f) $0 \mathrm{~min}$ (before injection) and (g) $70 \mathrm{~min}$ postinjection of PIGD NPs (2 mg/mL, $0.25 \mathrm{~mL}$ per rat). (h) PA amplitude and SNR (dB) as a function of postinjection time. The blue arrow indicates injection point. Laser energy density has been set at $\sim 5 \mathrm{~mJ} / \mathrm{cm}^{2}$. Reproduced with permission from Ref. 136. The wPAT for brain imaging of behaving rats: (i) photograph of the miniaturized 1064-nm wPAT probe, (j) and (k) PA images obtained at different dorsoventral planes in a behaving rat. Reproduced with permission from Ref. 153.

surface. For comparison, brain images were shown at both 750 [Fig. 6(d)] and $1064 \mathrm{~nm}$ [Fig. 6(e)]. The SNR at $1064 \mathrm{~nm}$ $(27.7 \pm 4.1 \mathrm{~dB})$ was 1.5 -fold enhanced than that at $750 \mathrm{~nm}$ $(18.2 \pm 4.9 \mathrm{~dB})$. These results confirmed the advantage of NIR-II over NIR-I PAI.

The PIGD nanoparticles (discussed in Sec. 3.1) were also demonstrated for in vivo brain imaging at $1064 \mathrm{~nm} .{ }^{136}$ The cerebral cortex of a rat brain was imaged at $1064 \mathrm{~nm}$ before and after a single injection of PIGD NPs $(0.25 \mathrm{~mL}$ per rat, $2 \mathrm{mg} / \mathrm{mL})$. The cross-sectional images of the rat brain at $0 \mathrm{~min}$ (before injection) and $70 \mathrm{~min}$ after injection are shown in Figs. 6(f) and $6(\mathrm{~g})$, respectively. The PA signal and image SNR as a function of postinjection time are shown in Fig. 6(h). The SNR of the image acquired at 0 min was $\sim 18 \mathrm{~dB}$ and it increased to $\sim 37 \mathrm{~dB}$ at $70 \mathrm{~min}$, i.e., an approximately twofold enhancement at 70-min postinjection. After $70 \mathrm{~min}$, the PA signal remained 
strong, indicating that a sufficient amount of NPs circulate in the blood. Long circulation of NPs could be due to their suitable size of $\sim 25 \mathrm{~nm}$ and the PEGylated surfaces. This in vivo brain imaging proved that even though blood absorption is low in the NIR-II window, by using suitable long-circulating nanoparticles, it is possible to achieve contrast-enhanced PAI of brain vessels of small animals with intact skin and skull. Several rat/mouse brain imaging works at $1064 \mathrm{~nm}$ using agents can be found in Refs. 154 and 155. Without the contrast agent, the cross section of a relatively large animal (monkey) brain cortex with skull thickness of $\sim 2 \mathrm{~mm}$ was imaged at $1064 \mathrm{~nm}$ with energy density $50 \mathrm{~mJ} / \mathrm{cm}^{2} .{ }^{156}$ With a strong 1064-nm agent, PAT can potentially image through even thicker skull bones. These results suggest that the NIR-II PAT system can potentially image human brain cortex in infants or even adults; however, more studies are needed in this direction.

Interestingly, a wearable scanning PAI (wPAI) system capable of detecting $\mathrm{HbO}, \mathrm{HbR}$, and $\mathrm{HbT}$ changes within the rat brain vasculatures under hyperoxia by using 1064,710 , and $797 \mathrm{~nm}$ wavelengths was reported. ${ }^{157}$ Later a 1064-nm wPAI was demonstrated for dorsoventral brain images noninvasively in behaving rats. ${ }^{153}$ Figure 6(i) shows the photograph of a 1064-nm wPAI probe. The 1064-nm beam was weakly converged and overlapped with the acoustic detection of a $40-\mathrm{MHz}$ microtransducer, which was attached to a cantilever. Figures 6(j) and $6(\mathrm{k})$ present wPAI images at positions of Bregma $-7.3 \mathrm{~mm}$ and Bregma $-2 \mathrm{~mm}$. The PA images provided brain vasculature information beneath the skull. The system could provide $\sim 0.5-\mathrm{mm}$ lateral and $0.1-\mathrm{mm}$ axial resolutions, and $11-\mathrm{mm}$ imaging depth with 1064-nm light and 40-MHz UST. The findings suggested that wearable PAT (wPAT) can be used for studying pathological events such as stroke, brain ischemia, and seizure.

\subsection{Lymph Nodes Imaging on Rats}

Sentinel lymph node (SLN) mapping is an important task for the staging of breast cancer, especially to find out if metastasis has happened or not. PAT is widely used for SLN imaging. ${ }^{64,67,158}$ In vivo lymph node imaging at $1064 \mathrm{~nm}$ is performed on rats using copper sulfide $(\mathrm{CuS}) .{ }^{123}$ A single-element UST-scanning PAT system, as described in Ref. 145, is used for the SLN imaging. It includes a Nd:YAG laser $(1064 \mathrm{~nm}$ at $1000 \mathrm{~mJ}, 15 \mathrm{~ns}$, $10 \mathrm{~Hz}$, model: LS-2137) from Symphotic Tii, Camarillo, and an unfocused UST (V323/2.25 MHz, Panametrics). The incident laser energy density is kept below $\sim 100 \mathrm{~mJ} / \mathrm{cm}^{2}$. The signals are subsequently amplified by amplifiers (ZFL-500LN, MiniCircuits and 5072PR, Panametrics), bandpass filtered, and finally recorded using a DAQ card (CS14100, Gage Applied, Inc.).

A semiconductor $\mathrm{CuS}$ nanoparticles with absorption around $1064 \mathrm{~nm}$ is demonstrated for enhancing the SLN contrast in living mouse. After interstitial injection of CuS NPs, the rat lymph nodes of $\sim 12 \mathrm{~mm}$ below the skin are clearly visible. Figure 7 shows the PAT images of the axillary and brachial lymph nodes of a rat $24 \mathrm{~h}$ after interstitial injection of $\mathrm{CuS} \mathrm{NPs}$ into the front paw pad. The lymph nodes, located $12 \mathrm{~mm}$ below the skin surface, are clearly visualized [Fig. 7(a)], the corresponding photograph is shown in Fig. 7(b). In contrast, the axillary and (a)

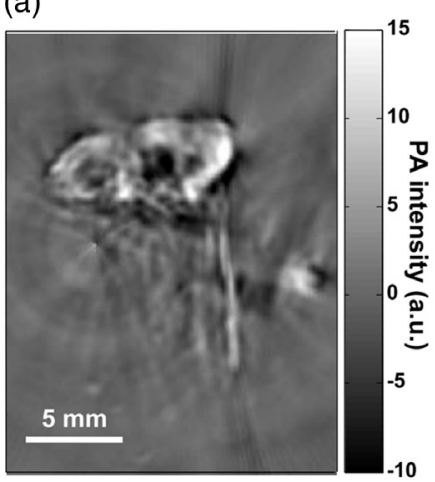

(c)

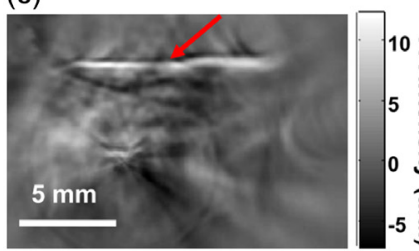

(b)

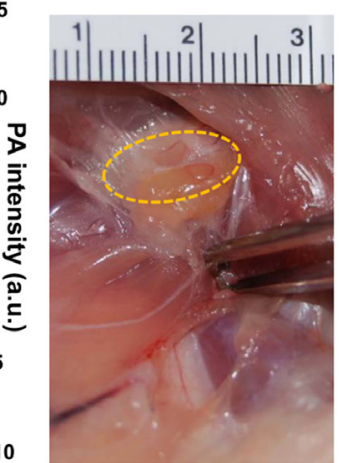

(d)

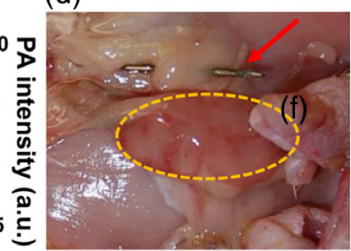

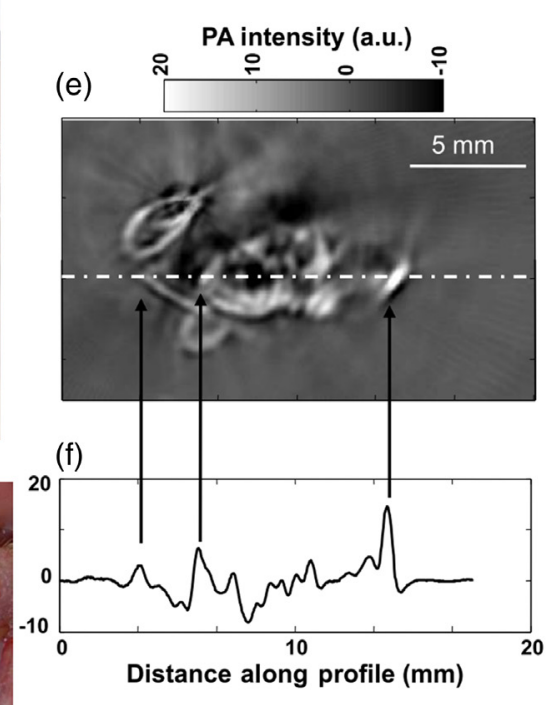

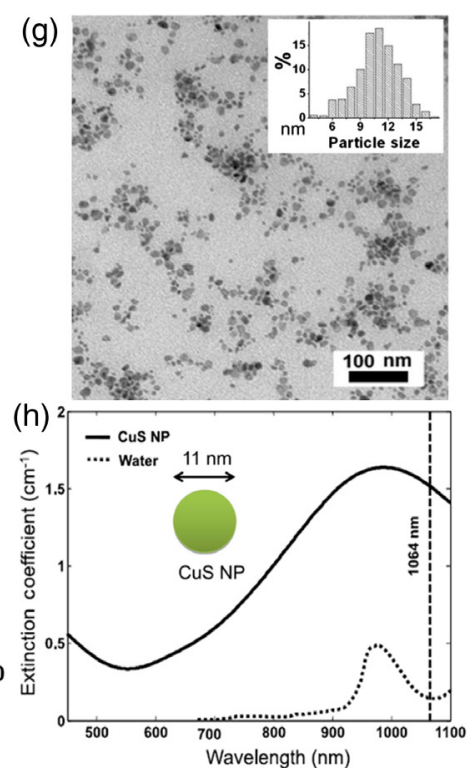

Fig. 7 Representative PAT images of axillary and brachial lymph nodes at a depth of $12 \mathrm{~mm}$ below the skin of rats: (a) PAT image acquired on the right side of a rat $24 \mathrm{~h}$ after interstitial injection of $200 \mu \mathrm{L}$ of CuS NP solution into the right front paw pad, (b) corresponding photograph of exposed rat underarm after imaging experiment, (c) PAT image acquired on the left side, into which no CuS NPs are injected, and (d) corresponding photograph of exposed rat underarm after imaging experiment. Yellow circles indicate lymph nodes. Red arrows indicate stainless steel needle tips placed adjacent to the lymph nodes to ensure that they are within the imaging field of view. (e) Representative PAT image of the axillary and brachial lymph nodes in a different rat. (f) One-dimensional profile showing PA signal intensity along the dashed line in (e). (g) TEM photograph of CuS NPs (inset: size distribution of CuS NPs). (h) Extinction coefficient spectra of 0.5-mM CuS NP aqueous solution (solid line) and pure water (dotted line). Reproduced with permission from Ref. 123. 
brachial lymph nodes on the contralateral side, which did not receive an interstitial injection of $\mathrm{CuS} \mathrm{NP}$, are not visualized on PAT [Fig. 7(c), its photograph is in Fig. 7(d). To ensure that the lymph nodes are within the imaging field of view, stainless steel needle tips are placed adjacent to the targets as a reference [red arrows on Figs. 7(c) and 7(d)]. PAT images in all rats depict the uptake of CuS NPs by ipsilateral draining lymph nodes. A representative lymph node image and its one-dimensional PA signal profile are shown in Figs. 7(e) and 7(f), respectively. The PA signal intensity is significantly higher in lymph nodes containing $\mathrm{CuS}$ NPs $(7.85 \pm 3.78)$ than in lymph nodes without $\mathrm{CuS}$ NPs $(2.46 \pm 0.73)$. TEM image of CuS NPs (average size $\sim 11 \mathrm{~nm}$ ) and the extinction coefficient spectrum (peaked around $990 \mathrm{~nm}$ ) of $0.5 \mathrm{mM} \mathrm{CuS}$ are shown in Figs. $7(\mathrm{~g})$ and 7(h), respectively. The imaging of the lymph node located at $\sim 12-\mathrm{mm}$ depth in a living rat promises that NIR-II PAT in combination with 1064-nm absorbing CuS nanoparticles could potentially be used for in vivo lymph node imaging in humans; however, clinical studies are needed to establish this.

\subsection{Canine Prostate Imaging}

Real-time visualization of regions of interest during surgery and biopsy procedures is important for clinicians. Clinically established modalities, such as MRI, PET, and CT allow whole-body scans and are ideal for diagnostic purposes. Owing to their very low-imaging speed, they are not suitable for real-time applications. Currently, US imaging is used for image-guided treatments. However, it is often difficult to deliver miniature probes to the surgical site without sacrificing the image quality. PAI has the potential to enable real-time visualization of regions of interest during surgery ${ }^{159-162}$ and biopsy. ${ }^{163,164}$ A 1064-nm side-firing fiber prototype for transurethral PAI of prostates with a dual-array (4-8 MHz/linear and 5-9 MHz/curvilinear) is reported. ${ }^{165}$ The design and photograph of the 1064-nm sidefiring fiber are shown in Figs. 8(a) and 8(b), respectively. The CT image of a BTS implanted in the canine prostate is shown in Fig. 8(c). The seed is implanted in the apex of the prostate and is imaged with US, as shown in Fig. 8(d); the arrow points to the BTS. The corresponding PA image [Fig. 8(e)], overlaid on the US image, contains the seed and the signals in the urethra and associated reverberation artifacts (likely caused by the metal tip). These artifacts are eliminated in Fig. 8(f). Thus, the BTS implanted in in vivo canine prostates at radial distances of 5 to $30 \mathrm{~mm}$ from the urethra are imaged with the fiber prototype transmitting 1064-nm wavelength light with 2- to 8-mJ pulse energy. It can display images in real time at a rate of 3 to 5 frames per second to guide fiber placement and beamformed offline.

\subsection{Skin and Tumor Imaging}

PAI of skin was performed in the visible region. ${ }^{166-168}$ Recently, skin and tumor imaging on small animals was demonstrated using the thienoisoindigo-based semiconducting polymer nanoparticles (TSPNs) [Fig. 9(a)]. It has strong absorption in 800 to $1350 \mathrm{~nm}$ [Fig. 9(b)] for PAI in the NIR-II window. ${ }^{169}$ An US imaging system (Vantage128, Verasonics Inc.) with EKSPLA OPO laser $(5 \mathrm{~ns}$ and $10 \mathrm{~Hz}$ ) was used for imaging. A refection-mode detection was applied using a high-frequency US array (L22-14v, Verasonics Inc.). For skin imaging [Fig. 9(c)], TSPNs $(50 \mu \mathrm{L}, 40 \mu \mathrm{g} / \mathrm{mL})$ were injected subcutaneously into the dorsal area of the rats. Without injection, the PA signal was weak at 1100 and $1300 \mathrm{~nm}$ and strong at 800 and $1000 \mathrm{~nm}$, due to the absorption of blood at these wavelengths. The strong signal at $1200 \mathrm{~nm}$ was generated from the subcutaneous fat in the skin layer. After injection, the PA signal increased by $\sim 7.3$-fold at $1100 \mathrm{~nm}$ and by $\sim 10.7$-fold at $1300 \mathrm{~nm}$, compared to the signal found in the skin tissue without injection. This significant signal enhancement indicated the capability of TSPN as an exogenous contrast agent for in vivo skin imaging.

In vivo images of tumor in mice with and without TSPNs are shown in Fig. 9(d). TSPNs are directly injected into PC3-M xenografts in mice. Without TSPNs, relatively strong PA signals are

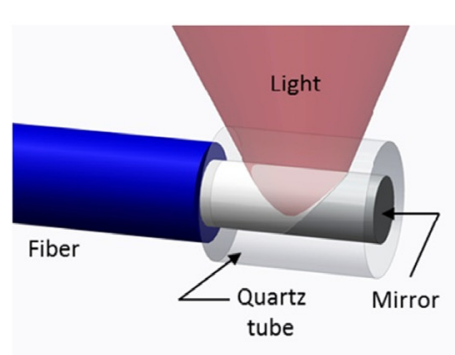

(a)

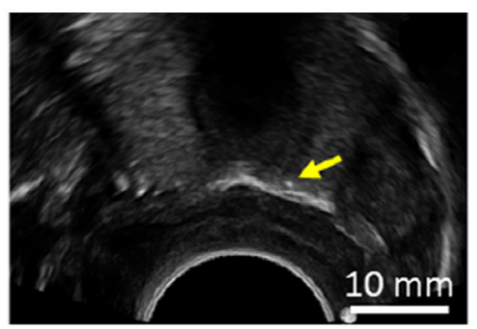

(d)

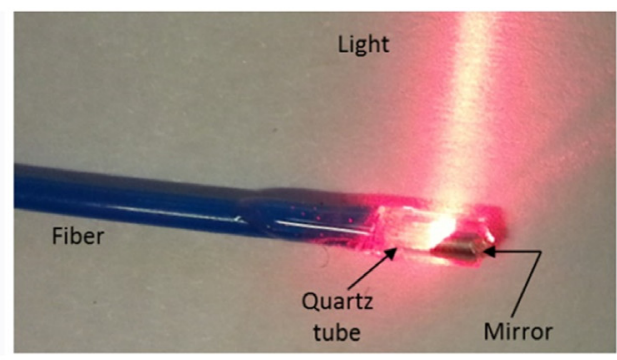

(b)

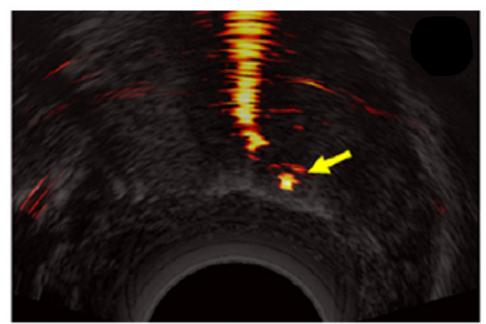

(e)

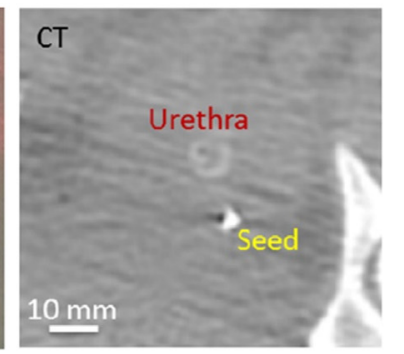

(c)

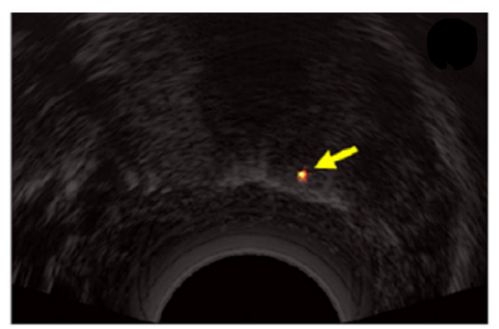

(f)

Fig. 8 (a) Design components of the 1064-nm side-firing fiber. (b) Photograph of the fiber prototype. In vivo CT (c) US, (d) PA without mask, (e) PA with mask, and (f) images of a BTS in the apex of the canine prostate. The yellow arrow identifies the BTS. Reproduced with permission from Ref. 165. 
(a)

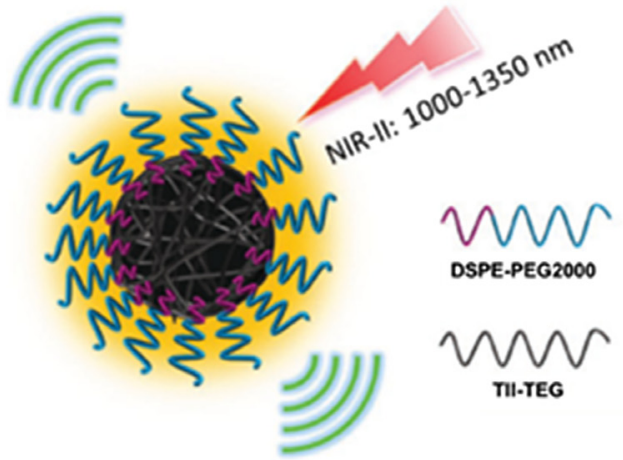

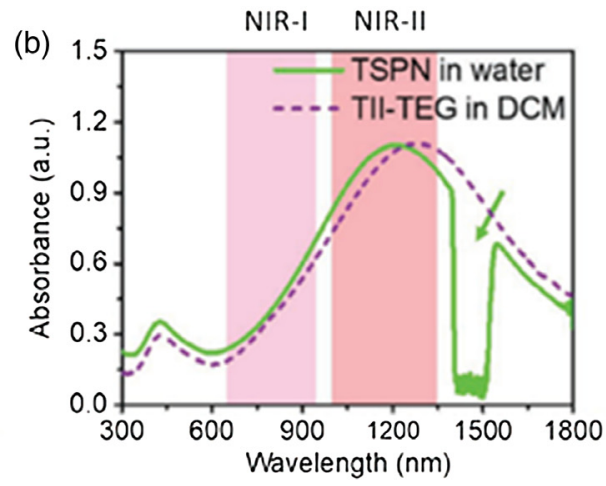

(c)

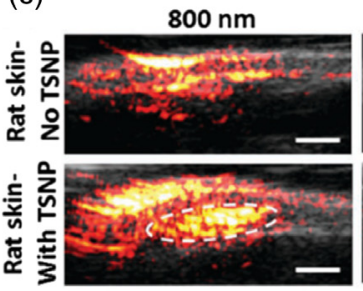

(d)
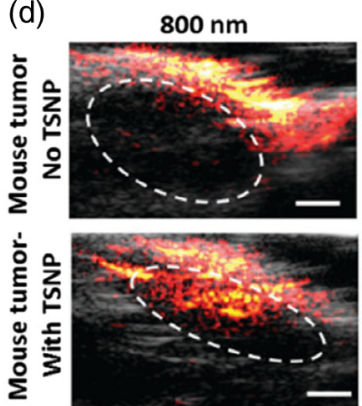

$1000 \mathrm{~nm}$
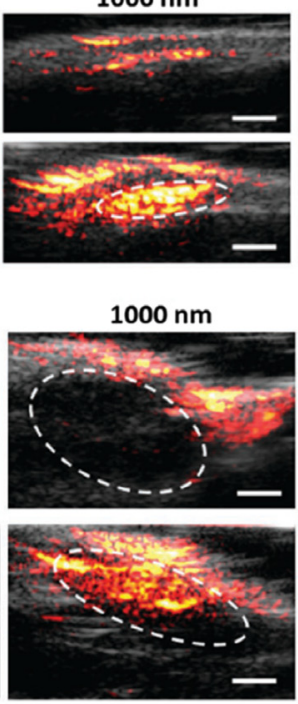
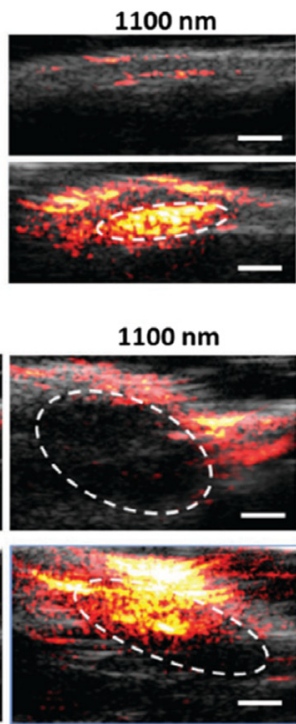

$1200 \mathrm{~nm}$

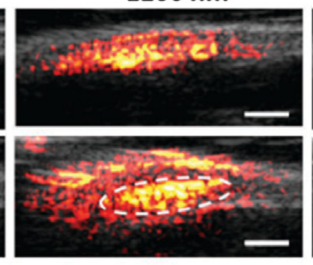

$1200 \mathrm{~nm}$

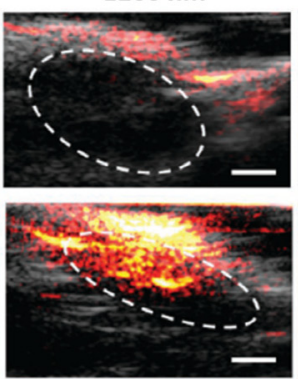

$1300 \mathrm{~nm}$

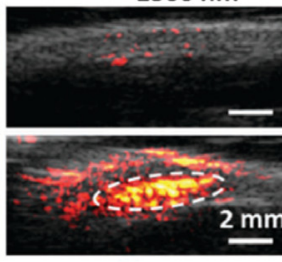

$1300 \mathrm{~nm}$

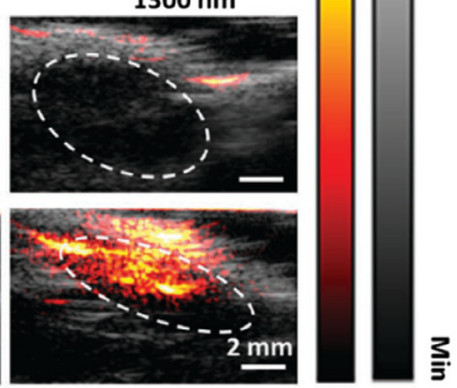

PA US

Fig. 9 (a) Schematic illustration of TSPNs prepared by nanoprecipitation. (b) Absorption spectra of TSPN in water (solid green line) and TII-TEG in dichloromethane (dashed purple line). The dip in the green curve is due to the strong absorption of water between 1400 and $1500 \mathrm{~nm}$. In vivo PA/US imaging of TSPN in rat skin (c) without injection of TSPNs (upper) and with injection of $50 \mu \mathrm{L}, 40 \mu \mathrm{g} / \mathrm{mL}$ matrigel inclusions of TSPNs (lower), and in mouse tumor (dashed circles); (d) without injection of TSPNs (upper) and with injection of $50 \mu \mathrm{L}, 40 \mu \mathrm{g} / \mathrm{mL}$ aqueous solution of TSPNs (lower). The laser energy density is $\sim 10 \mathrm{~mJ} / \mathrm{cm}^{2}$ at $800 \mathrm{~nm}, 19 \mathrm{~mJ} / \mathrm{cm}^{2}$ at $1000 \mathrm{~nm}, 33 \mathrm{~mJ} / \mathrm{cm}^{2}$ at $1100 \mathrm{~nm}, 37 \mathrm{~mJ} / \mathrm{cm}^{2}$ at $1200 \mathrm{~nm}$, and $25 \mathrm{~mJ} / \mathrm{cm}^{2}$ at $1300 \mathrm{~nm}$. Each image is normalized with the corresponding laser energy density. Reproduced with permission from Ref. 169.

observed from the tumor at 800 and $1000 \mathrm{~nm}$, which are attributed to the rich blood vessels surrounding the tumor. The signals above the tumor at $1200 \mathrm{~nm}$ are more likely from skin fats. Intratumoral injection was performed to deliver TSPNs $(50 \mu \mathrm{L}$, $40 \mu \mathrm{g} / \mathrm{mL}$ ) into the tumor area, then strong PA signals were generated within the tumor area (dashed circles) due to the strong absorption of excitation wavelengths 800 to $1300 \mathrm{~nm}$ by the TSPNs. Compared to the images of the tumor without TSPNs, the signal was enhanced $\sim 7.0$-fold at $1100 \mathrm{~nm}$ and $\sim 13.3$-fold at $1300 \mathrm{~nm}$. Reports on NIR-II tumor imaging ${ }^{116,154}$ promised that it can provide high sensitivity and depth for tumor imaging compared to NIR-I window.

\subsection{Urinary Bladder Imaging}

Urinary bladder imaging is critical to diagnose urinary tract disorders and bladder cancer. Recently, bladder imaging in rats has been shown with clinical US + PAI system. ${ }^{63}$ A clinical research US system (E-CUBE 12R, Alpinion, South Korea) that can acquire PA and US images simultaneously is used.
A linear array transducer (L3-12, 8.5 MHz, 95\% fractional bandwidth) with 128 elements is used for data acquisition. For PAI, 1064-nm light from a Nd:YAG pump laser $(10 \mathrm{~Hz}$, $5 \mathrm{~ns}$, Continuum, Surelite Ex) is transmitted through the bifurcated optical fiber bundle (Ceramoptec GmbH, Germany). The two rectangular fiber bundle output are fixed to the linear array transducer in such a manner that the fiber-to-transducer distance is $\sim 2 \mathrm{~cm}$, the fiber-to-tissue distance is $\sim 1 \mathrm{~cm}$, and a light delivery angle is at $15 \mathrm{deg}$. The energy on the rat skin is kept at $\sim 9 \mathrm{~mJ} / \mathrm{cm}^{2}$. Using this system, the structural (bladder wall), functional (accretion of gold nanoparticles), and the diagnosis of vesicoureteral reflux, a common bladder disorder, are successfully demonstrated in NIR-II window.

\subsection{Lipid Profile Imaging}

Lipid-based conditions appear in many diseases that include diabetes, obesity, fatty liver disease, and vascular diseases, such as coronary artery disease and peripheral artery disease. Optimum wavelengths for nerve or lipid imaging are 910, 1210, and 


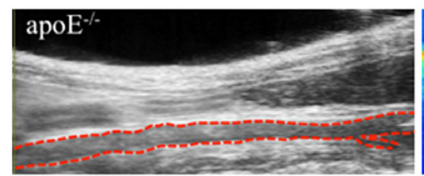

(a)

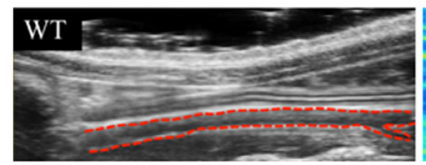

(d)

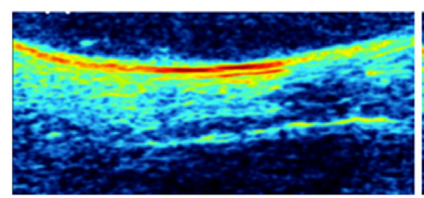

(g)

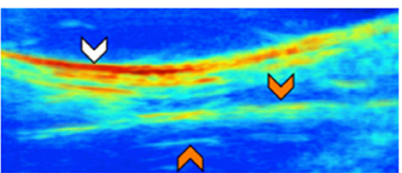

(b)

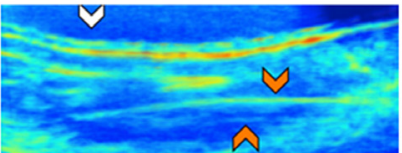

(e)

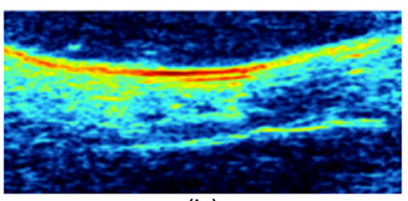

(h)

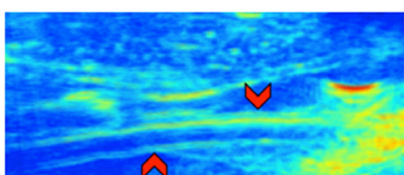

(c)

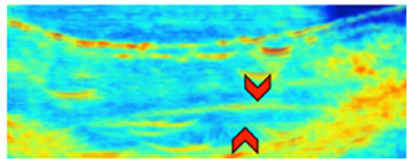

(f)

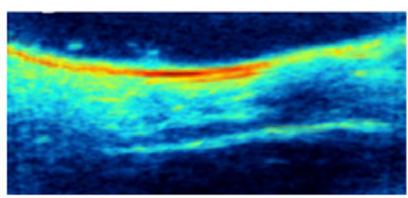

(i)

Fig. 10 US and PAT images of the adult male apoE-/- (a-c) and WT (d-f) mice. (b) and (e) Lipid PAT images at $1210 \mathrm{~nm}$; (c) and (f) blood PAT images at $1100 \mathrm{~nm}$. Mouse is orientated in supine position with the head located to the left and tail located to the right. Periaortic fat signal is more in the apoE-/- mouse compared to the WT mouse. The images can clearly discriminate the infrarenal aorta (red dotted outline), subcutaneous fat (white arrows), periaortic fat (orange arrows), and blood (red arrows). PAT raw images $(\mathrm{g}, \mathrm{h})$ and mean image (i) of an apoE-/-mouse at $1210 \mathrm{~nm}$. Reproduced with permission from Ref. 172.

$1700 \mathrm{~nm} .{ }^{170,171}$ Recently, in vivo murine lipid imaging is shown at $1210 \mathrm{~nm}$ on mice of different gender, genotypes, and maturation. ${ }^{172}$ The system used is an US system (Vevo2100, FUJIFILM Visual Sonics) equipped with a high-frequency transducer (MS550D, $40 \mathrm{MHz}$ ) to obtain PA + US images of the infrarenal aorta. A Nd:YAG/OPO laser (NT352C, Ekspla, 670 to $2300 \mathrm{~nm}, 5 \mathrm{~ns}, 10 \mathrm{~Hz}$ ) is used for lipid imaging. The US images of infrarenal aorta of apolipoprotein E-deficient (apoE-/-) and wild-type (WT) mice are shown in Figs. 10(a) and 10(d), respectively. The system resolves subcutaneous and periaortic fat buildup in both mice. The lipid-specific PAT signal follows both the geometry of the skin due to the subcutaneous fat and the infrarenal aorta due to periaortic fat accumulation. The adult male apoE-/- mice show more fat accumulation compared to the adult male WT mice. The raw [Figs. 10(g) and 10(h)] and averaged [Fig. 10(i)] PAT images at $1210 \mathrm{~nm}$ reveal subcutaneous and periaortic fat. In vivo lipid PAI is also reported at $1197 \mathrm{~nm} .{ }^{170}$ The light used for these studies is the low-energy idler light from OPO lasers. So, there is a need for high-pulse energy (hundreds of $\mathrm{mJ}$ ) laser with a lipidspecific wavelength of $910,1210,1700 \mathrm{~nm}$ and with a pulse repetition rate of at least $10-\mathrm{kHz}$ level for high-speed imaging.

\section{In Vivo Human Imaging}

In vivo human imaging is the ultimate goal. However, it is challenging. Whole-body MRI, x-ray CT, scintigraphy, PET CT scan, etc. are being used in clinics. The above-discussed deep-tissue and in vivo animal-imaging studies show promise that PAT has matured over the years and is progressing toward clinical use. This section discusses the advanced PAT systems operated at $1064 \mathrm{~nm}$ and their successful applications on humans for diagnosis purposes.

\subsection{Human Breast Imaging}

Breast cancer is the most common cancer among women. Symptoms include a lump or thickening of the breast and changes to the skin or the nipple. For breast cancer screening, mammography is still the gold standard. In recent times, MRI or even ultrasonography is being used as an alternative. Even the optical imaging modality, DOT, is used for monitoring neoadjuvant chemotherapy in patients with breast cancer. PACT has emerged as an alternative tool that overcomes many of the limitations associated with the existing modalities. ${ }^{173-175}$

A Twente photoacoustic mammoscope (Twente PAMa) based on a 1064-nm laser was developed [Fig. 11(a)] for human breast imaging. ${ }^{177,176}$ The PAMa included a Nd:YAG laser $(1064 \mathrm{~nm}, 10 \mathrm{~ns}, 10 \mathrm{~Hz}, 350 \mathrm{~mJ})$ from Continuum Surelite and an US array consisting of 588 elements $(1 \mathrm{MHz})$ in a circular layout with a diameter of $85 \mathrm{~mm}$. The 1064-nm beam was maintained at a fixed position on the breast surface, with a beam area of $\sim 35 \mathrm{~cm}^{2}$ and an energy density of $\sim 10 \mathrm{~mJ} / \mathrm{cm}^{2}$ on the breast skin. An imaging time of $10 \mathrm{~min}$ was required for the complete detector area covering a field of view of $90 \times 85 \mathrm{~mm}^{2}$ on the breast. Figures $11(\mathrm{~b})$ and $11(\mathrm{c})$ show the images in craniocaudal direction of MRI and PAT. The results showed that there is a good correspondence in lesion colocalization, appearance, and shape in the PAT and MRI images. The study has proven that PAI has potential in visualization of breast cancer. For the first time, mass-like, nonmass, and ring appearances were observed in PA images. A photoacoustic mammography system that can provide several wavelengths, such as 756, 797, 825, and $1064 \mathrm{~nm}$ (Canon Inc., Tokyo, Japan), was demonstrated for tumor morphology. ${ }^{178,179}$

Very recently, a single-breath-hold photoacoustic computed tomography (SBH-PACT) system, as shown in Fig. 11(d), was demonstrated for volumetric imaging of the human breast. ${ }^{36}$ The system used a 1064-nm light to achieve sufficient optical penetration in the breast tissue. The 1064-nm laser beam (PRO350-10, Quanta-Ray, $10 \mathrm{~Hz}, 8$ to $12 \mathrm{ns)} \mathrm{was} \mathrm{first} \mathrm{passed}$ through an axicon lens (25 mm, 160-deg apex angle), and was then expanded by an engineered diffuser (EDC-10-A-2 s, RPC Photonics) to form a donut-shaped light beam. The 1064-nm laser energy on the breast was $\sim 20 \mathrm{~mJ} / \mathrm{cm}^{2}$. To achieve a 2-D panoramic acoustic detection, a 512-element full-ring UST array (Imasonic, Inc., 220-mm ring diameter, $2.25 \mathrm{MHz}$ ) was 


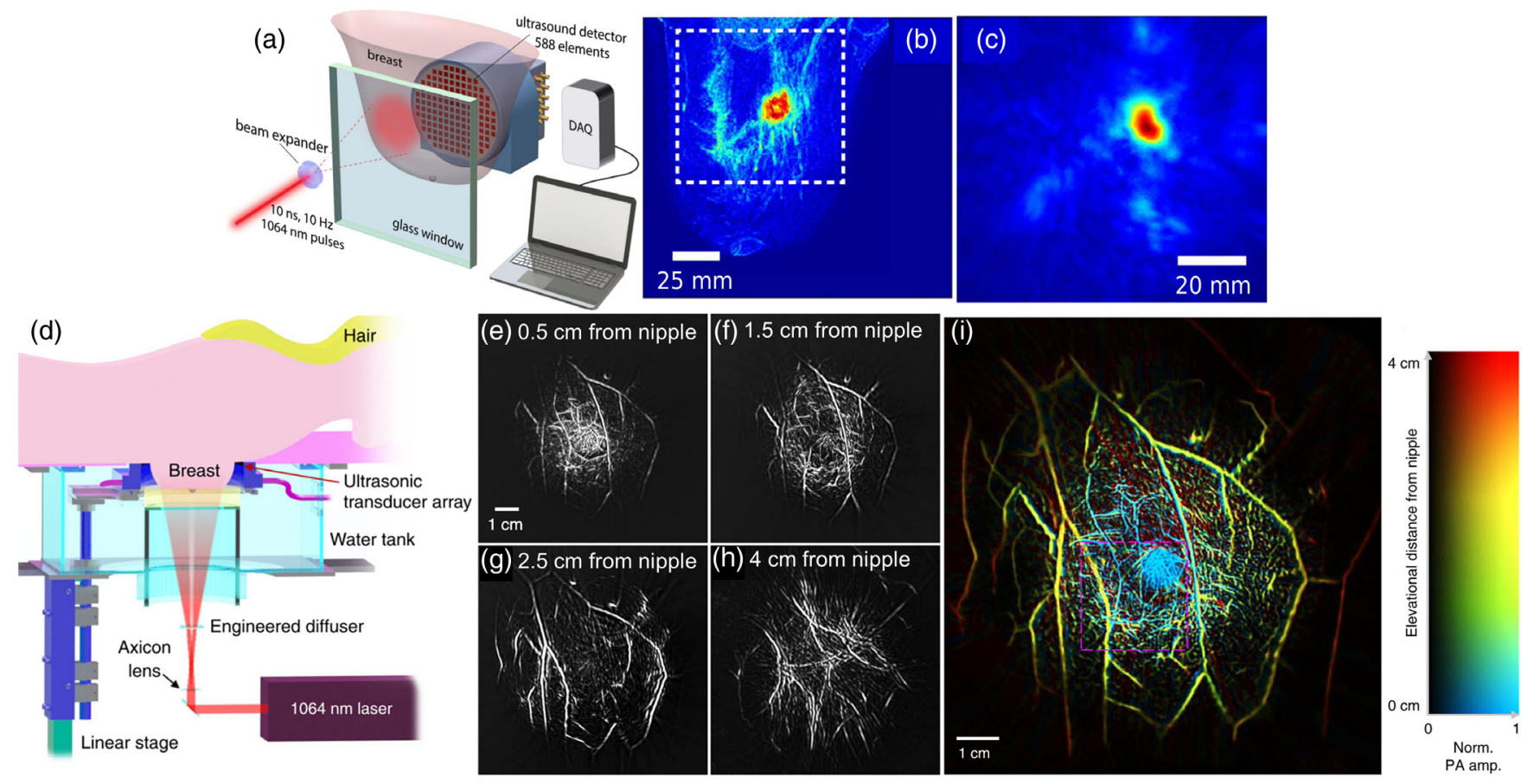

Fig. 11 (a) Schematic representations of the Twente PAMa. (b) MRI, and (c) PAI of the breast with tumor. These images were acquired from a patient having infiltrating ductal carcinoma. The dashed box in the MRI indicates the area from which the PA image was acquired. Reproduced with permission from Ref. 176. (d) Schematic representation of the SBH-PACT system: the laser illuminates the breast from beneath the patient's breast, and the UST array detects photoacoustic waves circumferentially around the breast. The light beam is converted into a donut shape via an axicon lens followed by an engineered diffuser. (e)-(h) Vasculature in the right breast of a 27-year-old healthy female volunteer. Normalized images at four depths are shown in increasing depth order from the nipple to the chest wall. (i) The same breast image with color-encoded depths. Reproduced with permission from Ref. 36.

employed. Four sets of 128-channel data acquisition systems provided simultaneous one-to-one mapped associations with the 512-element transducer array. A 128-channel data acquisition system (SonixDAQ, Ultrasonix Medical ULC, $40 \mathrm{MHz}$, 12 bit) with programmable amplification up to $51 \mathrm{~dB}$ was used. Therefore, time taken to acquire PA signals from a cross section was within $100 \mu$ s without multiplexing after each laser pulse excitation.

The performance of SBH-PACT was assessed by imaging a 27-year-old healthy female volunteer. By scanning the transducer array elevationally through her right breast, within one breath-hold $(\sim 15 \mathrm{~s})$, a volumetric image was acquired. The angiographic anatomy from the nipple to the chest wall was revealed. The breast images at different depth are shown in Figs. 11(e)-11(h). The color-encoded depth-resolved image clearly revealed the detailed angiographic structures of the entire breast [Fig. 11(i)], visualizing the vasculature down to an apparent vascular diameter of $258 \mu \mathrm{m}$. The system could provide a depth of $\sim 4 \mathrm{~cm}$, a spatial resolution at $\sim 255 \mu \mathrm{m}$, and a frame rate of $\sim 10 \mathrm{~Hz}$. Using hemoglobin as the contrast, SBH-PACT can potentially monitor breast cancer's response to neoadjuvant chemotherapy by acquiring information similar to that of contrast-enhanced MRI, yet with finer spatial resolution, higher imaging speed, and only endogenous contrast.

\subsection{Human Arm Imaging}

The second window is demonstrated for PAI of deep vasculature in human hand and palm. The system design used for arm/palm imaging is shown in Fig. 12(a). ${ }^{149}$ The system uses a compact 1064-nm laser (described in Sec. 4.2) and a bifurcated fiber bundle with circular input (1-cm diameter) and two line outputs (5-cm length) to deliver light to the arm/palm. The 1064-nm energy density used on the $5-\times 1-\mathrm{cm}$ skin surface is $8 \mathrm{~mJ} / \mathrm{cm}^{2}$ at $50 \mathrm{~Hz}$. For PA signal detection, an ATL/Philips L7-4 liner transducer array (elevation focus $25 \mathrm{~mm}$ and resolution $1.5 \mathrm{~mm}$ ) is used. During data acquisition, the object is kept stationary, and the transducer and fiber bundles scanned over a $75-\mathrm{mm}$ region at a speed of $1 \mathrm{~mm} / \mathrm{s}$. The system could scan 40-mm length in $8 \mathrm{~s}$, generating 4002 -D data sets. The raw-channel data are first filtered with a bandpass filter (5 to $8 \mathrm{MHz}$ ) and are then reconstructed with the backprojection algorithm. Photographs of the arm and palm are shown in Figs. 12(c) and $12(\mathrm{~d})$, respectively. The blood vessels are not visible in the photographs due to the strong tissue scattering in the visible region. In contrast, PA images in Figs. 12(d) and 12(e) clearly show blood vessels within the imaging region [red dashed box in Figs. 12(a) and 12(c)]. As expected, vascular distribution in the arm is much thinner than that of the palm. The SNR of the images is calculated using the selected signals (box 1 and box 3) and noises (box 2 and box 4). The SNR of the arm image is $39 \mathrm{~dB}$ and that of palm image is $36 \mathrm{~dB}$; no endogenous contrast agent has been used in this study.

Another 1064-nm PA/US system, as shown in Fig. 12(f), was demonstrated imaging through a human arm with highly absorbing contrast agent (P-Pc) at $1064 \mathrm{~nm} .{ }^{37}$ A tube containing the P-Pc formulation was placed beneath the arm of a healthy and conscious human adult. The arm was first imaged without 


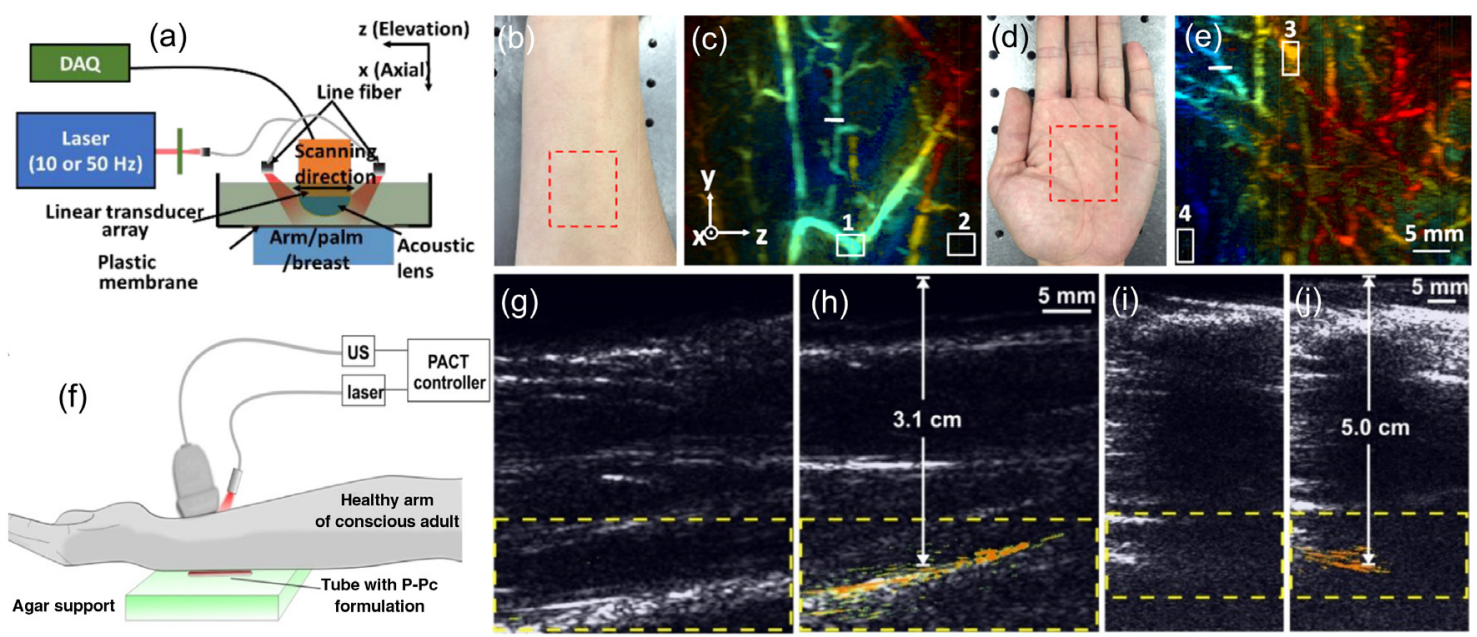

Fig. 12 In vivo arm and palm imaging of healthy human volunteers: (a) schematic representation of the setup used for palm imaging, (b) photograph of 25-year-old male human arm (red box indicates the imaged region), (c) depth-encoded maximum amplitude projection (MAP) image of the human arm. The $x, y$, and $z$ correspond to axial, lateral, and elevation directions of the transducer array, respectively. MAP is performed along the axial direction. (d) Photograph of 23-year-old male human palm (red box indicates the imaging region). (e) Depth-encoded MAP image of human palm. Reproduced with permission from Ref. 149. Translimb PACT of human arm using P-Pc: (f) experimental PACT setup, representative PA/US images of $(\mathrm{g}, \mathrm{h}) 3.1 \mathrm{~cm}$ and $(\mathrm{i}, \mathrm{j}) 5.0 \mathrm{~cm}$ arm. No P-Pc tube is present in (g) and (i), and P-Pc tube is present in (h) and (j) under the arm. The PA signal of the tube is clearly visible under the arm. The SNR is 37 and $32 \mathrm{~dB}$ in the 3.1- and 5.0-cm arm experiments, respectively. Reproduced with permission from Ref. 37.

the tube; the US could visualize the whole arm and no PA signal was present [Fig. 12(g)]. The US signal visible at the bottom of the arm corresponded to the US reflection from the supporting agar gel under the arm. However, when a tube containing P-Pc was placed under the arm, its complete shape could be visualized through the $3.1-\mathrm{cm}$ arm with an energy density of $\sim 14 \mathrm{~mJ} / \mathrm{cm}^{2}$ [Fig. 12(h)]. For another volunteer with a larger arm, the fluence used was $23 \mathrm{~mJ} / \mathrm{cm}^{2}$. The P-Pc tube was clearly detectable through the 5-cm arm [Figs. 12(i)-12(j)]. There was no bone visible in the imaging plane, because the transducer imaged in the gap between the ulna and the radius bones. Skin was not easily observed in the images because the transducer was placed directly on it.

\section{High-Resolution Photoacoustic Imaging in Near-Infrared-II Window}

Till now we have discussed the second window PAT/PACT systems and their deep-tissue (several centimeters) applications for live animal and human organ imaging. The usefulness of the second window is also demonstrated in high-resolution PAM imaging system. Typically in PAM the imaging depth is sacrificed to achieve higher resolution. For example, in optical resolution photoacoustic microscopy (OR-PAM), typical imaging depth up to 1 to $2 \mathrm{~mm}$ is obtained with lateral resolution of 0.25 to 5 micron, ${ }^{180,181}$ and in acoustic resolution microscopy (AR-PAM), 3 to $8 \mathrm{~mm}$ imaging depth is obtained with lateral resolution of 40 to 50 micron. ${ }^{182,183}$

Most of the PAM systems reported so far have used visible or NIR-I window due to the strong hemoglobin absorption. Recently, OR-PAM was reported for the first time in the second NIR window. ${ }^{113}$ The system includes a Nd:YLF/dye-laser $(1046,523$, and $570 \mathrm{~nm})$ and a 50-MHz UST. The OR-PAM images of a $50-\mu \mathrm{m}$-diameter black human hair obliquely inserted into a piece of fresh chicken breast tissue at $570 \mathrm{~nm}$ (visible), and $1046 \mathrm{~nm}$ (NIR-II) are shown in Figs. 13(a) and 13(b), respectively. The energy densities on the tissue surface at 570 and $1046 \mathrm{~nm}$ are 20 and $100 \mathrm{~mJ} / \mathrm{cm}^{2}$, respectively. A 1046-nm wavelength could image a 3.2-mm thick hair beneath the skin surface with a SNR of $6 \mathrm{~dB}$, whereas a 570-nm wavelength could image only up to $2.3-\mathrm{mm}$ depth with the same SNR. Similarly, the same area of the mouse brain is imaged at 570 [Fig. 13(c)] and $1046 \mathrm{~nm}$ [Fig. 13(d)]. At $570 \mathrm{~nm}$, blood vessels are clearly visible up to $0.25 \mathrm{~mm}$ and few vessels are visible up to $0.5 \mathrm{~mm}$ due to the strong blood absorption at $570 \mathrm{~nm}$. On the other hand, the 1046-nm wavelength could show vessels up to $0.75 \mathrm{~mm}$. The longer penetration and high spatial resolution in OR-PAM are due to the weaker optical attenuation and high ANSI limit of the NIR-II window.

Unlike OR-PAM, where tight optical focusing is used to achieve high resolution, in AR-PAM, lateral resolution is mainly dictated by the US focusing. As a result, imaging depth is increased to several millimeters with a compromised lateral resolution of 40 to 50 micron. All the AR-PAM systems reported so far use visible or NIR-I window. Very recently, a 1064-nm AR-PAM system has been reported for deep-tissue and in vivo imaging. ${ }^{184,185}$ A black tape embedded in a chicken breast tissue is imaged up to $11 \mathrm{~mm}$, as shown in Fig. 13(e). In vivo imaging of SLN [Fig. 13(f)] and urinary bladder [Fig. 13(g)] in rats using black ink as a contrast agent are reported. The system integrates a Nd:YAG $(1064 \mathrm{~nm})$ with $30-\mathrm{MHz}$ focused UST. Before injection, lymph node and urinary bladder are hardly visible. The black ink enhances the SLN and UB contrast, as shown in Figs. 13(f) and 13(g), respectively. The energy per pulse used for in vivo imaging is $1 \mathrm{~mJ} / \mathrm{cm}^{2}$. Thus, using NIR-II wavelengths further enhances the depth and resolution capabilities of PAM. 


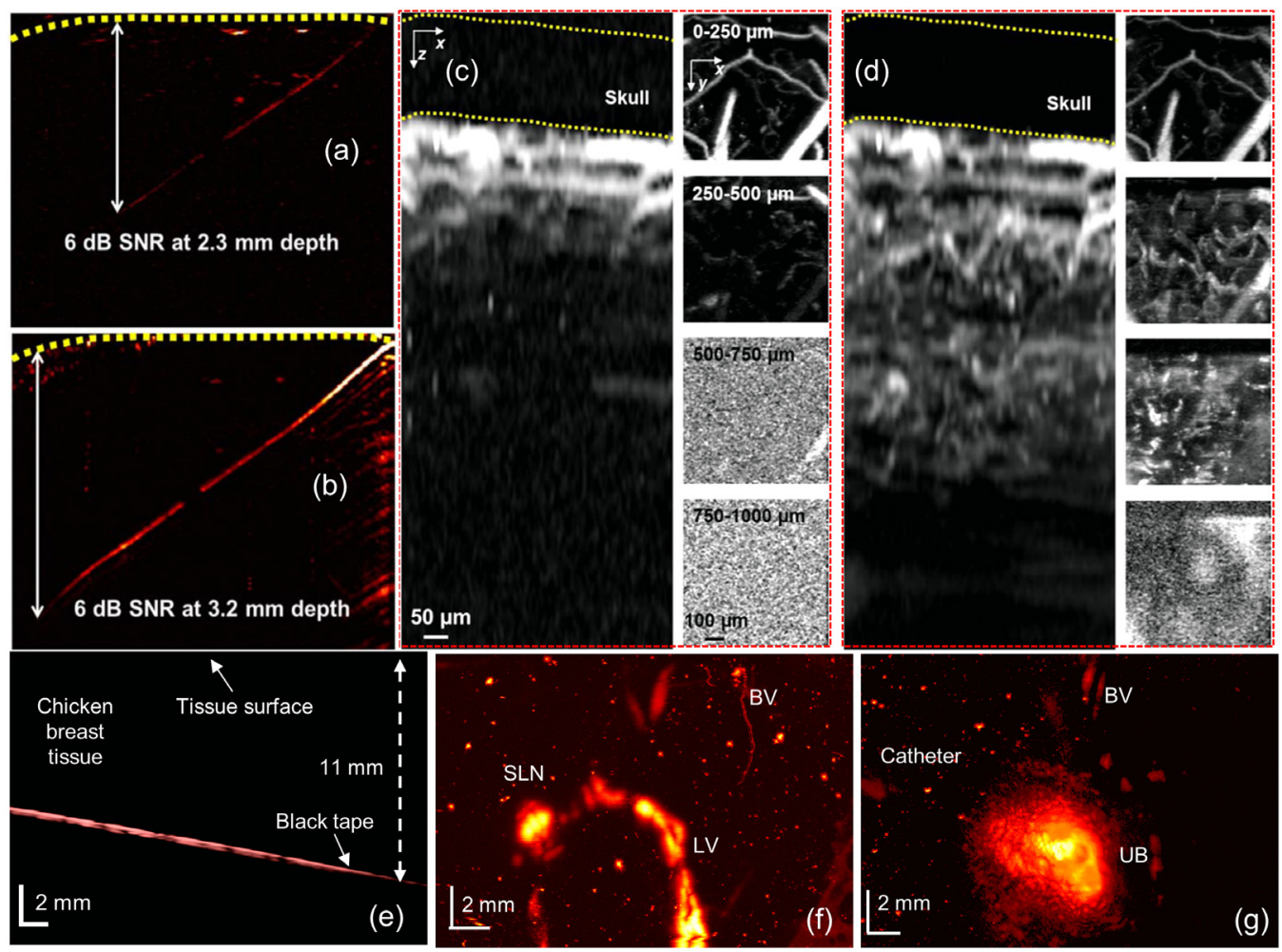

Fig. 13 OR-PAM images of a black human hair inserted obliquely into a piece of fresh chicken breast tissue: (a) image at $570 \mathrm{~nm}, 20 \mathrm{~mJ} / \mathrm{cm}^{2}$, (b) image at $1046 \mathrm{~nm}, 100 \mathrm{~mJ} / \mathrm{cm}^{2}$. Hair is imaged with a SNR of $\geq 6 \mathrm{~dB}$ up to $3.2-\mathrm{mm}$ deep in the tissue at 1046- and 2.3-mm deep in the tissue at $570 \mathrm{~nm}$. Comparison of in vivo imaging depths in a mouse brain: $x-z$ and $x-y$ projection images of the mouse brain at $570 \mathrm{~nm}$ (c) and at $1046 \mathrm{~nm}$ (d). At $570 \mathrm{~nm}$, no structure can be detected at depths beyond $500 \mu \mathrm{m}$. At $1046 \mathrm{~nm}$, structures can be detected at depths up to $750 \mu \mathrm{m}$. Reproduced with permission from Ref. 113. AR-PAM of black tape, SLN, and urinary bladder using black ink at $1064 \mathrm{~nm}$ : (e) B-scan of the black tape embedded in the chicken breast, (f) the SLN, lymph vessel (LV), and blood vessel (BV) are shown in the MAP PAI after black ink injection, (g) BV and urinary bladder are shown in the MAP PAI after the injection of black ink. Reproduced with permission from Ref. 184.

\section{Summary and Perspectives}

We have discussed the advanced PAT systems and contrast agents developed for NIR-II imaging. Numerous applications of the second window for in vitro studies, in vivo small animal imaging, and in vivo human imaging have been discussed. Imaging depth of up to $\sim 12 \mathrm{~cm}$ was demonstrated using P-Pc at $1064 \mathrm{~nm}$ at $56 \mathrm{~mJ} / \mathrm{cm}^{2} .{ }^{37}$ Imaging of the liver lesions was performed at $1064 \mathrm{~nm}$ using gold nanorods. ${ }^{124}$ Advantage of using the 1064-nm wavelength for brachytherapy has been shown by imaging a radioactive substance embedded $5-\mathrm{cm}$ deep inside a tissue phantom. ${ }^{138}$ In vivo skin imaging on rats was performed at 800,1000, 1200, and $1300 \mathrm{~nm}$, and lowest signal was observed at $1300 \mathrm{~nm} .{ }^{169}$ Although the above studies have used NIR-II agents, NIR-II window has shown its potential for PAI without an exogenous agent: in vivo small animal brain imaging and whole body of small animals using blood contrast at $1064 \mathrm{~nm} .{ }^{149,150}$ In vivo human imaging at $1064 \mathrm{~nm}$ was successfully demonstrated by imaging human palm/arm, ${ }^{149}$ and breast ${ }^{36}$ without a contrast agent. The cross section of the monkey brain cortex with a skull thickness of $\sim 2 \mathrm{~mm}$ was imaged at $1064 \mathrm{~nm}$ with energy density of $50 \mathrm{~mJ} / \mathrm{cm}^{2} .{ }^{156}$ The PAT images at $1210 \mathrm{~nm}$ revealed the lipid (subcutaneous fat, periaortic fat) profile along the anterior wall of the infrarenal aorta of mouse. ${ }^{172}$ These studies have proved that NIR-II PAT system operated at $1064 \mathrm{~nm}$ is a very promising tool for preclinical applications.

Twente PAMa is demonstrated for human breast imaging at $1064 \mathrm{~nm}$ with the energy density of $10 \mathrm{~mJ} / \mathrm{cm}^{2}$. The system could image the tumor inside the breast but cannot provide blood vessel information inside and around the tumor due to its poor resolution $(\sim 3.5 \mathrm{~mm})$. Imaging speed of this system is very low (10 min/image) and hence is not suitable for dynamic imaging. ${ }^{176}$ The SBH-PACT, an advanced three-dimensional breast imaging system using $1064 \mathrm{~nm}$, is a promising tool for both clinical screening and diagnostic studies. ${ }^{36}$ It offers 4-cm depth (1064 $\mathrm{nm}$ at $\left.20 \mathrm{~mJ} / \mathrm{cm}^{2}\right)$ to image the whole breast, $255-\mu \mathrm{m}$ high resolution to reveal blood vessels in and around tumor, and $10-\mathrm{Hz}$ high frame rate for dynamic imaging. No exogenous contrasts have been used in both Twente PAM and SBH-PACT for breast imaging. This proves the clinical potential of $1064 \mathrm{~nm}$ PACT for human imaging applications.

To compensate for the reduced blood (an intrinsic contrast agent) absorption in NIR-II region, several NIR-II absorbing agents were developed. Here, we have listed out some of the agents and the wavelength of maximum absorbance in the second window: (i) NIR-II inorganic contrast agents-singlewalled carbon nanotubes (1064 $\left.\mathrm{nm}^{126}\right), \mathrm{CuS}$ nanoparticles $\left(990 \mathrm{~nm}^{123}\right)$, Ag nanoplates $\left(1050 \mathrm{~nm}^{125}\right)$, $\mathrm{Au}$ nanorods $\left(1040 \mathrm{~nm}^{124}\right.$ and $\left.1064 \mathrm{~nm}^{186}\right)$, silica-coated gold nanoplates 
$\left(1064 \mathrm{~nm}^{122}\right)$, Au plasmonic blackbody $\left(1000 \mathrm{~nm}^{119}\right)$, metalorganic particles $\left(1064 \mathrm{~nm}^{120}\right), \mathrm{Bi}_{2} \mathrm{Se}_{3}$ nanoplates $\left(1064 \mathrm{~nm}^{121}\right)$; (ii) NIR-II organic contrast agents-P-Pc $\left(1000 \mathrm{~nm}^{37}\right)$, organic charge-transfer nanocomplex $\left(\sim 1000 \mathrm{~nm}^{116}\right)$, semiconducting nanoparticles $\left(1064 \mathrm{~nm}^{118}, 1253 \mathrm{~nm},{ }^{152} 1200 \mathrm{~nm},{ }^{169} 960 \mathrm{~nm}^{136}\right.$, and $1079 \mathrm{~nm}{ }^{187}$ ), etc. Though they have broad spectrum, all these nanoparticles were demonstrated for PAI at $1064 \mathrm{~nm}$ due to the availability of high-energy pulsed laser at this wavelength.

Although these exciting studies have shown the potential of NIR-II window for preclinical and clinical PAI, the NIR-II imaging is still in its infancy. Like any other newly developed imaging technology, second window imaging also has some fundamental and technological challenges: (i) NIR-II contrast agents with excellent biocompatibility and favorable clearance profiles similar to those of the clinically approved NIR-I small molecule dyes (ICG, methylene blue, Evans blue) are needed; (ii) biocompatibility, pharmacokinetics, and long-term toxicity of NIR-II contrast agents is not sufficient; (iii) tunable yet powerful NIR-II pulsed laser is not available; (iv) optical properties of biological tissues required for fundamental studies need to be identified in the NIR-II region. The NIR-II contrast agent synthesis studies have focused on developing nanomaterials with low quantum yield (increasing the nonradiative transitions) to enhance their PA efficiency. But the major challenge revolves around the elucidation of the biocompatibility, pharmacokinetics, and long-term toxicological profile of the NIR-II agents, which are crucial for medical applications. ${ }^{188}$ There is a potential leakage of heavy metal ions from inorganic NIR-II contrast agents. Some of the NIR-II organic nanoparticles, such as SPNs, have long circulation time; they stay several days in the body. For NIR-II imaging, there is no FDA-approved small molecule for clinical use. P-Pc is a small molecule that could readily be formulated in commonly used surfactants to produce solutions with extreme absorption beyond $1000 \mathrm{~nm}$. The P-Pc is used for PAI through $11.6 \mathrm{~cm}$ of chicken breast tissue. Although this molecule is used in tissue phantoms, the deep detection of P-Pc through over $10 \mathrm{~cm}$ of biological tissue shows its potential for clinical applications. However, still more research work needs to be done to develop an ideal NIR-II PA contrast agent that shows fast and complete clearance from the body, high photothermal conversion efficiency, and low cost.

Another challenge is developing a tunable yet powerful NIR-II laser. Although the NIR-II contrast agents show broad absorption, they are demonstrated for PAI at $1064 \mathrm{~nm}$ due to the availability of 1064-nm high-energy pulsed lasers in laboratory/market. The applications of the single-wavelength PAT are limited to structural imaging. It is challenging to extract functional information with a single-wavelength excitation; usually two or more wavelengths are needed. ${ }^{189}$ If the absorption peak of the contrast agent does not match with the excitation wavelength (e.g., $1064 \mathrm{~nm}$ ), its real efficiency cannot be demonstrated. So there is an urgent need for developing a high-energy nanosecond laser tunable in the NIR-II window. The commercial Vevo LAZR-X multimodal PA/US system is incorporated with a laser source that can deliver 680 to $970 \mathrm{~nm}$ (NIR-I) and 1200 to $2000 \mathrm{~nm}$ (NIR-II). ${ }^{190}$ However, nanosecond lasers that can provide energy density as high as $100 \mathrm{~mJ} / \mathrm{cm}^{2}$ or even more are needed to fully utilize the high MPE of the second window. The idler wavelengths of commercial Nd:YAG/OPO laser fall in the NIR-II region, but their energy levels (which are very low compared to NIR-I window) and beam quality makes it not ideal for the second window imaging. Fundamental studies on light penetration in biological tissue at different wavelengths are still needed for quantitative image analysis. The large variations in the tissue composition across different organs and different individuals makes it difficult to carry out quantitative studies. The applications of NIR-II PAT are still at the early stage; most of them are proof-ofconcept studies, and so more complex imaging applications, including functional imaging, molecular imaging, ${ }^{24}$ metabolism imaging, ${ }^{191}$ biosensing, ${ }^{192}$ and human disease ${ }^{193-195}$ need to be demonstrated.

\section{Conclusion}

Overall, the above studies have proved that, compared to the NIR-I window, the NIR-II window offers longer penetration depth and high contrast imaging due to the (1) reduced tissue scattering, (2) minimal tissue absorption, (3) higher MPE offered by the NIR-II window, and (4) lower cost. Shifting the excitation window from NIR-I to NIR-II has significantly enhanced the performance of PAI systems by improving the depth and image quality. By employing fast scanning technologies, multiple wavelengths, miniaturized laser technology, efficient yet safe contrast agents, and by combining with existing modalities, PAT can become a promising tool for clinical screening, diagnosis, and therapy applications in the near future.

\section{Disclosures}

Authors have no relevant financial interests in the manuscript and no other potential conflicts of interest to disclose.

\section{Acknowledgments}

The authors would like to acknowledge the financial support from the Singapore Ministry of Health's National Medical Research Council (NMRC/OFIRG/0005/2016: M4062012).

\section{References}

1. S. H. Yun and S. J. J. Kwok, "Light in diagnosis, therapy and surgery," Nat. Biomed. Eng. 1(1), 0008 (2017).

2. P. K. Upputuri et al., "Recent developments in vascular imaging techniques in tissue engineering and regenerative medicine," BioMed Res. Int. 2015, 1-9 (2015).

3. A. R. Kherlopian et al., "A review of imaging techniques for systems biology," BMC Syst. Biol. 2, 74 (2008).

4. A. J. Beer and M. Schwaiger, "Imaging of integrin alphavbeta3 expression," Cancer Metastasis Rev. 27(4), 631-644 (2008).

5. H. Degani et al., "Magnetic resonance imaging of tumor vasculature," Thromb. Haemost. 89(1), 25-33 (2003).

6. D. Wu and J. Zhang, "In vivo mapping of macroscopic neuronal projections in the mouse hippocampus using high-resolution diffusion MRI," Neuroimage 125, 84-93 (2016).

7. M. Sarracanie et al., "Low-cost high-performance MRI," Sci. Rep. 5, 15177 (2015).

8. D. J. Brenner and E. J. Hall, "Computed tomography—an increasing source of radiation exposure," N. Engl. J. Med. 357, 2277-2284 (2007).

9. A. Greco et al., "Ultrasound biomicroscopy in small animal research: applications in molecular and preclinical imaging," J. Biomed. Biotechnol. 2012, 1-14 (2012).

10. B. C. Wilson, M. Jermyn, and F. Leblond, "Challenges and opportunities in clinical translation of biomedical optical spectroscopy and imaging," J. Biomed. Opt. 23(3), 030901 (2018).

11. D. Nguyen et al., "Optical projection tomography for rapid whole mouse brain imaging," Biomed. Opt. Express 8(12), 5637-5650 (2017).

12. G. C. Kagadis et al., "In vivo small animal imaging: current status and future prospects," Med. Phys. 37(12), 6421-6442 (2010). 
13. A. V. DSouza et al., "Review of fluorescence guided surgery systems: identification of key performance capabilities beyond indocyanine green imaging," J. Biomed. Opt. 21(8), 80901 (2016).

14. J.-L. H. Demers et al., "Next-generation Raman tomography instrument for non-invasive in vivo bone imaging," Biomed. Opt. Express 6(3), 793 (2015)

15. P. K. Upputuri, L. Gong, and H. Wang, "Chirped time-resolved CARS microscopy with square-pulse excitation," Opt. Express 22(8), 9611-9626 (2014).

16. E. M. Hillman et al., "In vivo optical imaging and dynamic contrast methods for biomedical research," Philos. Trans. A Math. Phys. Eng. Sci. 369(1955), 4620-4643 (2011).

17. L. V. Wang and J. Yao, "A practical guide to photoacoustic tomography in the life sciences," Nat. Methods 13(8), 627-638 (2016).

18. P. K. Upputuri et al., "Super-resolution coherent anti-Stokes Raman scattering microscopy with photonic nanojets," Opt. Express 22(11), 12890-12899 (2014).

19. X. Shu, L. J. Beckmann, and H. F. Zhang, "Visible-light optical coherence tomography: a review," J. Biomed. Opt. 22(12), 121707 (2017).

20. R. Raghunathan et al., "Optical coherence tomography for embryonic imaging: a review," J. Biomed. Opt. 21(5), 050902 (2016).

21. B. J. Tromberg et al., "Assessing the future of diffuse optical imaging technologies for breast cancer management," Med. Phys. 35(6), 2443-2451 (2008).

22. R. Choe et al., "Diffuse optical tomography of breast cancer during neoadjuvant chemotherapy: a case study with comparison to MRI," Med. Phys. 32(4), 1128-1139 (2005).

23. J. E. Gunther et al., "Dynamic diffuse optical tomography for monitoring neoadjuvant chemotherapy in patients with breast cancer," Radiology 287(3), 778-786 (2018).

24. J. Yao and L. V. Wang, "Recent progress in photoacoustic molecular imaging," Curr. Opin. Chem. Biol. 45, 104-112 (2018).

25. P. K. Upputuri and M. Pramanik, "Recent advances toward preclinical and clinical translation of photoacoustic tomography: a review," J. Biomed. Opt. 22(4), 041006 (2017).

26. S. Manohar and D. Razansky, "Photoacoustics: a historical review," Adv. Opt. Photonics 8(4), 586-617 (2016).

27. Y. Zhou, J. Yao, and L. V. Wang, "Tutorial on photoacoustic tomography," J. Biomed. Opt. 21(6), 061007 (2016).

28. M. Mozaffarzadeh et al., "Eigenspace-based minimum variance combined with delay multiply and sum beamforming algorithm: application to linear-array photoacoustic imaging," IEEE J. Sel. Top. Quant. Electron. 25(1), 1-8 (2019).

29. S. Gutta et al., "Deep neural network-based bandwidth enhancement of photoacoustic data," J. Biomed. Opt. 22(11), 116001 (2017).

30. S. K. Kalva and M. Pramanik, "Experimental validation of tangential resolution improvement in photoacoustic tomography using a modified delay-and-sum reconstruction algorithm," J. Biomed. Opt. 21(8), 086011 (2016).

31. M. Pramanik, "Improving tangential resolution with a modified delayand-sum reconstruction algorithm in photoacoustic and thermoacoustic tomography," J. Opt. Soc. Am. A 31(3), 621-627 (2014).

32. C. B. Shaw et al., "Least squares QR-based decomposition provides an efficient way of computing optimal regularization parameter in photoacoustic tomography," J. Biomed. Opt. 18(8), 080501 (2013).

33. N. A. Rejesh, H. Pullagurla, and M. Pramanik, "Deconvolution-based deblurring of reconstructed images in photoacoustic/thermoacoustic tomography," J. Opt. Soc. Am. A 30(10), 1994-2001 (2013).

34. B. Cox et al., "Quantitative spectroscopic photoacoustic imaging: a review," J. Biomed. Opt. 17(6), 061202 (2012).

35. B. E. Treeby, E. Z. Zhang, and B. T. Cox, "Photoacoustic tomography in absorbing acoustic media using time reversal," Inverse Prob. 26(11), 115003 (2010).

36. L. Lin et al., "Single-breath-hold photoacoustic computed tomography of the breast," Nat. Commun. 9(1), 2352 (2018).

37. Y. Zhou et al., "A phosphorus phthalocyanine formulation with intense absorbance at $1000 \mathrm{~nm}$ for deep optical imaging," Theranostics 6(5), 688-697 (2016).

38. L. V. Wang and S. Hu, "Photoacoustic tomography: in vivo imaging from organelles to organs," Science 335(6075), 1458-1462 (2012).

39. X. Deán-Ben et al., "Advanced optoacoustic methods for multiscale imaging of in vivo dynamics," Chem. Soc. Rev. 46(8), 2158-2198 (2017).
40. D. Razansky, A. Buehler, and V. Ntziachristos, "Volumetric real-time multispectral optoacoustic tomography of biomarkers," Nat. Protoc. 6(8), 1121-1129 (2011).

41. B. Park et al., "Super-resolution photoacoustic microscopy using nearfield localization by a plasmonic metal nanoaperture: a simulation study," IEEE J. Sel. Top. Quant. Electron. 25(2), 1-7 (2019).

42. P. K. Upputuri, M. Krishnan, and M. Pramanik, "Microsphere enabled sub-diffraction limited optical-resolution photoacoustic microscopy: a simulation study," J. Biomed. Opt. 22(4), 045001 (2017).

43. S. Lee et al., "Super-resolution visible photoactivated atomic force microscopy," Light: Sci. Appl. 6(11), e17080 (2017).

44. A. Danielli et al., "Label-free photoacoustic nanoscopy," J. Biomed. Opt. 19(8), 086006 (2014).

45. P. K. Upputuri et al., "Super-resolution photoacoustic microscopy using photonic nanojets: a simulation study," J. Biomed. Opt. 19(11), 116003 (2014).

46. J. Yao et al., "Photoimprint photoacoustic microscopy for three-dimensional label-free subdiffraction imaging," Phys. Rev. Lett. 112(1), 014302 (2014).

47. T. Chuangsuwanich et al., "Photoacoustic imaging of lamina cribrosa microcapillary in porcine eyes," Appl. Opt. 57(17), 4865-4871 (2018).

48. T. J. Allen et al., "Large area laser scanning optical resolution photoacoustic microscopy using a fibre optic sensor," Biomed. Opt. Express 9(2), 650-660 (2018).

49. K. Park et al., "Handheld photoacoustic microscopy probe," Sci. Rep. 7(1), 13359 (2017).

50. M. Moothanchery et al., "In vivo studies of transdermal nanoparticle delivery with microneedles using photoacoustic microscopy," Biomed. Opt. Express 8(12), 5483-5492 (2017).

51. K. Sathiyamoorthy, E. M. Strohm, and M. C. Kolios, "Low-power noncontact photoacoustic microscope for bioimaging applications," J. Biomed. Opt. 22(4), 046001 (2017).

52. N. G. Horton et al., "In vivo three-photon microscopy of subcortical structures within an intact mouse brain," Nat Photonics 7(3), 205-209 (2013).

53. K. Xiong et al., "Autofocusing optical-resolution photoacoustic endoscopy," Opt. Lett. 43(8), 1846-1849 (2018).

54. Y. Li et al., "In vivo photoacoustic/ultrasonic dual-modality endoscopy with a miniaturized full field-of-view catheter," J. Biophotonics 11(10), e201800034 (2018).

55. H. He et al., "Hybrid optical and acoustic resolution optoacoustic endoscopy," Opt. Lett. 41(12), 2708 (2016).

56. J.-M. Yang et al., "Simultaneous functional photoacoustic and ultrasonic endoscopy of internal organs in vivo," Nat. Med. 18(8), 1297-1302 (2012).

57. S. K. Kalva, P. K. Upputuri, and M. Pramanik, "High-speed, low-cost, pulsed-laser-diode-based second-generation desktop photoacoustic tomography system," Opt. Lett. 44(1), 81-84 (2019).

58. A. Sharma, S. K. Kalva, and M. Pramanik, "A comparative study of continuous versus stop-and-go scanning in circular scanning photoacoustic tomography," IEEE J. Sel. Top. Quantum Electron. 25(1), 1-9 (2019).

59. D. M. Egolf, R. K. W. Chee, and R. J. Zemp, "Sparsity-based reconstruction for super-resolved limited-view photoacoustic computed tomography deep in a scattering medium," Opt. Lett. 43(10), 2221-2224 (2018).

60. Y. Qu et al., "Dichroism-sensitive photoacoustic computed tomography," Optica 5(4), 495-501 (2018).

61. S. K. Kalva, Z. Z. Hui, and M. Pramanik, "Calibrating reconstruction radius in a multi single-element ultrasound-transducer-based photoacoustic computed tomography system," J. Opt. Soc. Am. A 35(5), 764-771 (2018).

62. P. K. Upputuri et al., "A high-performance compact photoacoustic tomography system for in vivo small-animal brain imaging," J. Visual. Exp. 124, e55811 (2017).

63. K. Sivasubramanian et al., "Hand-held, clinical dual mode ultrasoundphotoacoustic imaging of rat urinary bladder and its applications," J. Biophotonics 11(5), e201700317 (2018).

64. K. Sivasubramanian, V. Periyasamy, and M. Pramanik, "Non-invasive sentinel lymph node mapping and needle guidance using clinical handheld photoacoustic imaging system in small animal," J. Biophotonics 11(1), e201700061 (2018). 
65. K. Sivasubramanian et al., "Optimizing light delivery through fiber bundle in photoacoustic imaging with clinical ultrasound system: Monte Carlo simulation and experimental validation," J. Biomed. Opt. 22(4), 041008 (2017).

66. J. Kim et al., "Programmable real-time clinical photoacoustic and ultrasound imaging system," Sci. Rep. 6, 35137 (2016).

67. A. Garcia-Uribe et al., "Dual-modality photoacoustic and ultrasound imaging system for noninvasive sentinel lymph node detection in patients with breast cancer," Sci. Rep. 5, 15748 (2015).

68. K. Daoudi et al., "Handheld probe integrating laser diode and ultrasound transducer array for ultrasound/photoacoustic dual modality imaging," Opt. Express 22(21), 26365-26374 (2014).

69. X. D. Wang et al., "Three-dimensional laser-induced photoacoustic tomography of mouse brain with the skin and skull intact," Opt. Lett. 28(19), 1739-1741 (2003).

70. D. K. Yao et al., "Optimal ultraviolet wavelength for in vivo photoacoustic imaging of cell nuclei," J. Biomed. Opt. 17(5), 056004 (2012).

71. A. Fleck and D. Begg, "The estimation of ribonucleic acid using ultraviolet absorption measurements," Biochim. Biophys. Acta, Nucl. Acids Protein Synth. 108(3), 333-339 (1965).

72. G. Ku and L. V. Wang, "Deeply penetrating photoacoustic tomography in biological tissues enhanced with an optical contrast agent," Opt. Lett. 30(5), 507-509 (2005).

73. G. Lu and B. Fei, "Medical hyperspectral imaging: a review," J. Biomed. Opt. 19(1), 010901 (2014).

74. H. Kang et al., "Real-time functional optical-resolution photoacoustic microscopy using high-speed alternating illumination at 532 and 1064 nm," J. Biophotonics 11(3), e201700210 (2018).

75. A. P. Jathoul et al., "Deep in vivo photoacoustic imaging of mammalian tissues using a tyrosinase-based genetic reporter," Nat. Photonics 9(4), 239-246 (2015).

76. P. K. Upputuri and M. Pramanik, "Pulsed laser diode based optoacoustic imaging of biological tissues," Biomed. Phys. Eng. Express 1(4), 045010-045017 (2015).

77. L. Xiang et al., "4-D photoacoustic tomography," Sci. Rep. 3, 1113 (2013).

78. D. Das et al., "On-chip generation of microbubbles in photoacoustic contrast agents for dual modal ultrasound/photoacoustic in vivo animal imaging," Sci. Rep. 8(1), 6401 (2018).

79. K. Sivasubramanian et al., "Near infrared light-responsive liposomal contrast agent for photoacoustic imaging and drug release applications," J. Biomed. Opt. 22(4), 041007 (2017).

80. J. Zhang et al., "Activatable photoacoustic nanoprobes for in vivo ratiometric imaging of peroxynitrite," Adv. Mater. 29(6), 1604764 (2017).

81. Y. Gawale et al., "Carbazole linked NIR Aza-BODIPY dyes as triplet sensitizers and photoacoustic contrast agents for deep tissue imaging," Chem. - A Eur. J. 23(27), 6570-6578 (2017).

82. Y. Jiang and K. Pu, "Advanced photoacoustic imaging applications of near-infrared absorbing organic nanoparticles," Small 13(30), 1700710 (2017).

83. S. Huang et al., "A dual-functional benzobisthiadiazole derivative as an effective theranostic agent for near-infrared photoacoustic imaging and photothermal therapy," J. Mater. Chem. B 4(9), 1696-1703 (2016).

84. M. Mathiyazhakan et al., "In situ synthesis of gold nanostars within liposomes for controlled drug release and photoacoustic imaging," Sci. China Mater. 59(11), 892-900 (2016).

85. J. Weber, P. C. Beard, and S. E. Bohndiek, "Contrast agents for molecular photoacoustic imaging," Nat. Methods 13(8), 639-650 (2016).

86. C. Wiraja et al., "Near-infrared light-sensitive liposomes for enhanced plasmid DNA transfection," Bioeng. Transl. Med. 1(3), 357-364 (2016).

87. C. Kim, C. Favazza, and L. V. Wang, "In vivo photoacoustic tomography of chemicals: high-resolution functional and molecular optical imaging at new depths," Chem. Rev. 110(5), 2756-2782 (2010).

88. M. Pramanik et al., "In vivo carbon nanotube-enhanced non-invasive photoacoustic mapping of the sentinel lymph node," Phys. Med. Biol. 54(11), 3291-3301 (2009).

89. J. Brunker et al., "Photoacoustic imaging using genetically encoded reporters: a review," J. Biomed. Opt. 22(7), 070901 (2017).

90. P. K. Upputuri and M. Pramanik, "Fast photoacoustic imaging systems using pulsed laser diodes: a review," Biomed. Eng. Lett. 8(2), 167-181 (2018).
91. P. K. Upputuri and M. Pramanik, "Dynamic in vivo imaging of small animal brain using pulsed laser diode-based photoacoustic tomography system," J. Biomed. Opt. 22(9), 090501 (2017).

92. P. K. Upputuri and M. Pramanik, "Performance characterization of low-cost, high-speed, portable pulsed laser diode photoacoustic tomography (PLD-PAT) system," Biomed. Opt. Express 6(10), 4118-4129 (2015).

93. R. G. M. Kolkman, W. Steenbergen, and T. G. van Leeuwen, "In vivo photoacoustic imaging of blood vessels with a pulsed laser diode," Lasers Med. Sci. 21(3), 134-139 (2006).

94. J. S. Allen and P. Beard, "Pulsed near-infrared laser diode excitation system for biomedical photoacoustic imaging," Opt. Lett. 31(23), 3462-3464 (2006).

95. W. Xia et al., "Handheld real-time LED-based photoacoustic and ultrasound imaging system for accurate visualization of clinical metal needles and superficial vasculature to guide minimally invasive procedures," Sensors 18(5), 1394 (2018).

96. Y. Zhu et al., "Light emitting diodes based photoacoustic imaging and potential clinical applications," Sci. Rep. 8(1), 9885 (2018).

97. A. Hariri et al., "The characterization of an economic and portable LED-based photoacoustic imaging system to facilitate molecular imaging," Photoacoustics 9, 10-20 (2018).

98. X. Dai, H. Yang, and H. Jiang, "In vivo photoacoustic imaging of vasculature with a low-cost miniature light emitting diode excitation," Opt. Lett. 42(7), 1456-1459 (2017).

99. T. J. Allen and P. C. Beard, "Light emitting diodes as an excitation source for biomedical photoacoustics," Proc SPIE 8581, 85811F (2013).

100. R. S. Hansen, "Using high-power light emitting diodes for photoacoustic imaging," Proc SPIE 7968, 79680A (2011).

101. "American National Standard for Safe Use of Lasers," ANSI Standard Z136.1-2007, NY (2007).

102. F. Gao et al., "Titania-coated 2D gold nanoplates as nanoagents for synergistic photothermal/sonodynamic therapy in the second nearinfrared window," Nanoscale 11, 2374-2384 (2019).

103. P. Wang et al., "Fabrication of red blood cell-based multimodal theranostic probes for second near-infrared window fluorescence imagingguided tumor surgery and photodynamic therapy," Theranostics $\mathbf{9}(2)$, 369-380 (2019).

104. Kenry, Y. Duan, and B. Liu, "Recent advances of optical imaging in the second near-infrared window," Adv. Mater 30(47), 1802394 (2018).

105. Z. Ma et al., "A theranostic agent for cancer therapy and imaging in the second near-infrared window," Nano Res. 12(2), 273-279 (2018).

106. A. L. Antaris et al., "A small-molecule dye for NIR-II imaging," Nat. Mater. 15(2), 235-242 (2016).

107. L. A. Sordillo et al., "Deep optical imaging of tissue using the second and third near-infrared spectral windows," J. Biomed. Opt. 19(5), 056004 (2014).

108. A. M. Smith, M. C. Mancini, and S. Nie, "Second window for in vivo imaging," Nat Nanotechnol. 4(11), 710-711 (2009).

109. A. A. Oraevsky et al., "Optoacoustic imaging of blood for visualization and diagnostics of breast cancer," Proc SPIE 4618, 81-94 (2002).

110. Y. Jiang and K. Pu, "Molecular fluorescence and photoacoustic imaging in the second near-infrared optical window using organic contrast agents," Adv. Biosyst. 2(5), 1700262 (2018).

111. Y. Liu et al., "Imaging molecular signatures for clinical detection of scleroderma in the hand by multispectral photoacoustic elastic tomography," J. Biophotonics 11(6), e201700267 (2018).

112. K. S. Valluru, K. E. Wilson, and J. K. Willmann, "Photoacoustic imaging in oncology: translational preclinical and early clinical experience," Radiology 280(2), 332-349 (2016).

113. P. Hai et al., "Near-infrared optical-resolution photoacoustic microscopy," Opt. Lett. 39(17), 5192-5195 (2014).

114. R. H. Silverman et al., "High-resolution photoacoustic imaging of ocular tissues," Ultrasound Med. Biol. 36(5), 733-742 (2010).

115. R. I. Siphanto et al., "Serial noninvasive photoacoustic imaging of neovascularization in tumor angiogenesis," Opt. Express 13(1), 89-95 (2005).

116. Z. Wang et al., "pH-sensitive and biodegradable charge-transfer nanocomplex for second near-infrared photoacoustic tumor imaging," Nano Res. 12(1), 49-55 (2019). 
117. Z. Cao et al., "Semiconducting polymer-based nanoparticles with strong absorbance in NIR-II window for in vivo photothermal therapy and photoacoustic imaging," Biomaterials 155, 103-111 (2018).

118. B. Guo et al., "Biocompatible conjugated polymer nanoparticles for highly efficient photoacoustic imaging of orthotopic brain tumors in the second near-infrared window," Mater. Horiz. 4(5), 1151-1156 (2017).

119. J. Zhou et al., "Compact plasmonic blackbody for cancer theranosis in near-infrared II window," ACS Nano 12(3), 2643-2651 (2018).

120. D. Zhang et al., "Self-quenched metal-organic particles as dual-mode therapeutic agents for photoacoustic imaging-guided second nearinfrared window photochemotherapy," ACS Appl. Mater. Interfaces 10(30), 25203-25212 (2018).

121. S. Park et al., "Bi2Se3 Nanoplates for contrast-enhanced photoacoustic imaging at $1064 \mathrm{~nm}, "$ Nanoscale 10(44), 20548-20558 (2018).

122. G. P. Luke et al., "Silica-coated gold nanoplates as stable photoacoustic contrast agents for sentinel lymph node imaging," Nanotechnology 24(45), 455101 (2013).

123. G. Ku et al., "Copper sulfide nanoparticles as a new class of photoacoustic contrast agent for deep tissue imaging at $1064 \mathrm{~nm}$," ACS Nano 6(8), 7489-7496 (2012).

124. Y. S. Chen et al., "Feasibility of contrast-enhanced photoacoustic liver imaging at a wavelength of $1064 \mathrm{~nm}$," in Biomed. Opt. and $3 D$ Imaging OSA, OSA, Miami, FL (2012).

125. K. Homan et al., "Prospects of molecular photoacoustic imaging at 1064 nm wavelength," Opt. Lett. 35(15), 2663-2669 (2010).

126. M. Pramanik et al., "Single-walled carbon nanotubes as a multimodalthermoacoustic and photoacoustic-contrast agent," J. Biomed. Opt. 14(3), 034018 (2009).

127. G. Hong, A. L. Antaris, and H. Dai, "Near-infrared fluorophores for biomedical imaging," Nat. Biomed. Eng. 1(1), 0010 (2017).

128. S. Jacques and S. Prahl, "Oregon Medical Laser Center," https://omlc .org/spectra/index.html (1 September 2018).

129. A. N. Yaroslavsky et al., "Optical properties of selected native and coagulated human brain tissues in vitro in the visible and near infrared spectral range," Phys. Med. Biol. 47(12), 2059-2073 (2002).

130. V. G. Peters et al., "Optical properties of normal and diseased human breast tissues in the visible and near infrared," Phys. Med. Biol. 35(9), 1317-1334 (1990)

131. K. Huang et al., "Nanomaterials for photoacoustic imaging in second near-infrared window," Biomater. Sci. 7(2), 472-479 (2019).

132. S. He et al., "Crucial breakthrough of second near-infrared biological window fluorophores: design and synthesis toward multimodal imaging and theranostics," Chem. Soc. Rev. 47(12), 4258-4278 (2018).

133. G. Hong et al., "Carbon nanomaterials for biological imaging and nanomedicinal therapy," Chem. Rev. 115(19), 10816-10906 (2015).

134. S. L. Jacques, "Optical properties of biological tissues: a review," Phys. Med. Biol. 58(11), R37-R61 (2013).

135. A. N. Bashkatov, E. A. Genina, and V. V. Tuchin, "Optical properties of skin, subcutaneous, and muscle tissues: a review," J. Innov. Opt. Heal Sci. 4(1), 9-38 (2011).

136. P. K. Upputuri et al., "Contrast-enhanced photoacoustic imaging in the second near-infrared window using semiconducting polymer nanoparticles," J. Biomed. Opt. 24(3), 031002 (2018).

137. H. Ke et al., "Performance characterization of an integrated ultrasound, photoacoustic, and thermoacoustic imaging system," J. Biomed. Opt. 17(5), 056010 (2012).

138. T. Harrison and R. J. Zemp, "Coregistered photoacoustic-ultrasound imaging applied to brachytherapy," J. Biomed. Opt. 16(8), 080502 (2011).

139. J. Xia and L. V. Wang, "Small-animal whole-body photoacoustic tomography: a review," IEEE Trans. Biomed. Eng. 61(5), 1380-1389 (2014).

140. D. Wang, Y. Wu, and J. Xia, "Review on photoacoustic imaging of the brain using nanoprobes," Neurophotonics 3(1), 010901 (2016).

141. J. Yao and L. V. Wang, "Photoacoustic brain imaging: from microscopic to macroscopic scales," Neurophotonics 1(1), 011003 (2014).

142. $\mathrm{L}$. Li et al., "Label-free photoacoustic tomography of whole mouse brain structures ex vivo," Neurophotonics 3(3), 035001 (2016).

143. S. Hu, "Listening to the brain with photoacoustics," IEEE J. Sel. Top. Quant. Electron. 22(3), 117-126 (2016).

144. X. Yang et al., "Photoacoustic tomography of small animal brain with a curved array transducer," J. Biomed. Opt. 14(5), 054007 (2009).
145. X. Wang et al., "Noninvasive photoacoustic angiography of animal brains in vivo with near-infrared light and an optical contrast agent," Opt. Lett. 29(27), 730-702 (2004).

146. T. F. Fehm et al., "In vivo whole-body optoacoustic scanner with real-time volumetric imaging capacity," Optica 3(11), 1153-1159 (2016).

147. J. Xia et al., "Whole-body ring-shaped confocal photoacoustic computed tomography of small animals in vivo," J. Biomed. Opt. 17(5), 050506 (2012).

148. R. Ma et al., "Multispectral optoacoustic tomography (MSOT) scanner for whole-body small animal imaging," Opt. Express 17(24), 2141421426 (2009).

149. D. Wang et al., "Deep tissue photoacoustic computed tomography with a fast and compact laser system," Biomed. Opt. Express 8(1), 112-123 (2017).

150. L. Li et al., "Single-impulse panoramic photoacoustic computed tomography of small-animal whole-body dynamics at high spatiotemporal resolution," Nat. Biomed. Eng. 1(1), 0071 (2017).

151. M. A. Anastasio et al., "Half-time image reconstruction in thermoacoustic tomography," IEEE Trans. Med. Imaging 24(2), 199-210 (2005).

152. Y. Jiang et al., "Broadband absorbing semiconducting polymer nanoparticles for photoacoustic imaging in second near-infrared window," Nano Lett. 17(8), 4964-4969 (2017).

153. J. Tang, X. Dai, and H. Jiang, "Wearable scanning photoacoustic brain imaging in behaving rats," J. Biophotonics 9(6), 570-575 (2016).

154. B. Guo et al., "Through scalp and skull NIR-II photothermal therapy of deep orthotopic brain tumors with precise photoacoustic imaging guidance," Adv. Mater. 30(35), 1802591 (2018).

155. P. Zhang et al., "High-resolution deep functional imaging of the whole mouse brain by photoacoustic computed tomography in vivo," J. Biophotonics 11(1), e201700024 (2017).

156. X. Yang and L. V. Wang, "Monkey brain cortex imaging by photoacoustic tomography," J. Biomed. Opt. 13(4), 044009 (2008).

157. J. Tang et al., "Noninvasive high-speed photoacoustic tomography of cerebral hemodynamics in awake-moving rats," J. Cereb. Blood Flow Metab. 35(8), 1224-1232 (2015).

158. K. H. Song et al., "Near-infrared gold nanocages as a new class of tracers for photoacoustic sentinel lymph node mapping on a rat model," Nano Lett. 9(1), 183-188 (2009).

159. J. Shubert and M. A. L. Bell, "Photoacoustic imaging of a human vertebra: implications for guiding spinal fusion surgeries," Phys. Med. Biol. 63(14), 144001 (2018).

160. B. Eddins and M. A. L. Bell, "Design of a multifiber light delivery system for photoacoustic-guided surgery," J. Biomed. Opt. 22(4), 041011 (2017).

161. N. Gandhi et al., "Photoacoustic-based approach to surgical guidance performed with and without a Da Vinci robot," J. Biomed. Opt. 22(12), 121606 (2017).

162. S. Kim et al., "Photoacoustic image guidance for robot-assisted skull base surgery," in IEEE Int. Conf. Rob. Autom. (ICRA) (2015).

163. J. Shubert and M. A. L. Bell, "Photoacoustic based visual Servoing of needle tips to improve biopsy on obese patients," in IEEE Int. Ultrason. Symp. (IUS) (2017).

164. D. Piras et al., "Photoacoustic needle: minimally invasive guidance to biopsy," J. Biomed. Opt. 18(7), 070502 (2013).

165. M. A. L. Bell et al., "Transurethral light delivery for prostate photoacoustic imaging," J. Biomed. Opt. 20(3), 036002 (2015).

166. M. Schwarz et al., "Optoacoustic Imaging of Skin," in Imaging in Dermatol., pp. 375-385 (2016).

167. D. Xu et al., "Noninvasive and high-resolving photoacoustic dermoscopy of human skin," Biomed. Opt. Express 7(6), 2095-2102 (2016).

168. C. P. Favazza, L. A. Cornelius, and L. V. Wang, "In vivo functional photoacoustic microscopy of cutaneous microvasculature in human skin," J. Biomed. Opt. 16(2), 026004 (2011).

169. J. Wu et al., "Semiconducting polymer nanoparticles for centimetersdeep photoacoustic imaging in the second near-infrared window," Adv. Mater. 29(41), 1703403 (2017).

170. J. Hui et al., "Bond-selective photoacoustic imaging by converting molecular vibration into acoustic waves," Photoacoustics 4(1), 11-21 (2016). 
171. T. P. Matthews et al., "Label-free photoacoustic microscopy of peripheral nerves," J. Biomed. Opt. 19(1), 016004 (2014).

172. G. S. Sangha, E. H. Phillips, and C. J. Goergen, "In vivo photoacoustic lipid imaging in mice using the second near-infrared window," Biomed. Opt. Express 8(2), 736-742 (2017).

173. T. T. Wong et al., "Fast label-free multilayered histology-like imaging of human breast cancer by photoacoustic microscopy," Sci. Adv. 3(5), e1602168 (2017).

174. M. Zhang et al., "Ultrasound-guided photoacoustic imaging for the selective detection of EGFR-expressing breast cancer and lymph node metastases," Biomed. Opt. Express 7(5), 1920-1931 (2016).

175. X. Li et al., "High resolution functional photoacoustic tomography of breast cancer," Med. Phys. 42(9), 5321-5328 (2015).

176. M. Heijblom et al., "Photoacoustic image patterns of breast carcinoma and comparisons with Magnetic Resonance Imaging and vascular stained histopathology," Sci. Rep. 5, 11778 (2015).

177. M. Heijblom et al., "The state of the art in breast imaging using the Twente Photoacoustic Mammoscope: results from 31 measurements on malignancies," Eur. Radiol. 26(11), 3874-3887 (2016).

178. Y. Asao et al., "Photoacoustic mammography capable of simultaneously acquiring photoacoustic and ultrasound images," J. Biomed. Opt. 21(11), 116009 (2016).

179. T. Kitai et al., "Photoacoustic mammography: initial clinical results," Breast Cancer 21(2), 146-153 (2014).

180. E. M. Strohm, M. J. Moore, and M. C. Kolios, "High resolution ultrasound and photoacoustic imaging of single cells," Photoacoustics 4(1), 36-42 (2016).

181. G. Ku et al., "Photoacoustic microscopy with 2-microm transverse resolution," J. Biomed. Opt. 15(2), 021302 (2011).

182. M. Moothanchery and M. Pramanik, "Performance characterization of a switchable acoustic and optical resolution photoacoustic microscopy system," Sensors 17(2), 357 (2017).

183. S. Park et al., "Acoustic resolution photoacoustic microscopy," Biomed. Eng. Lett. 4(3), 213-222 (2014).

184. V. Periyasamy et al., "1064 $\mathrm{nm}$ acoustic resolution photoacoustic microscopy," J. Biophotonics e201800357 (2019).

185. M. Moothanchery et al., "High resolution and deep tissue imaging using a near infrared acoustic resolution photoacoustic microscopy," Proc SPIE 10494, 104945R (2018).

186. Y. S. Chen et al., "Miniature gold nanorods for photoacoustic molecular imaging in the second near-infrared optical window," Nat. Nanotechnol. (2019).
187. Y. Jiang et al., "Metabolizable semiconducting polymer nanoparticles for second near-infrared photoacoustic imaging," Adv. Mater. 31(11), 1808166 (2019).

188. L. Nie and X. Chen, "Structural and functional photoacoustic molecular tomography aided by emerging contrast agents," Chem. Soc. Rev. 43(20), 7132-7170 (2014).

189. Y. Liang et al., " $2 \mathrm{MHz}$ multi-wavelength pulsed laser for functional photoacoustic microscopy," Opt. Lett. 42(7), 1452-1455 (2017).

190. A. Forbrich, A. Heinmiller, and R. J. Zemp, "Photoacoustic imaging of lymphatic pumping," J. Biomed. Opt. 22(10), 1-6 (2017).

191. J. J. Yao et al., "Noninvasive photoacoustic computed tomography of mouse brain metabolism in vivo," Neuroimage 64(1), 257-266 (2013).

192. B. S. Goldschmidt et al., "Photoacoustic measurement of refractive index of dye solutions and myoglobin for biosensing applications," Biomed. Opt. Express 4(11), 2463-2476 (2013).

193. Y. Matsumoto et al., "Label-free photoacoustic imaging of human palmar vessels: a structural morphological analysis," Sci. Rep. 8(1), 786 (2018).

194. M. Yang et al., "Photoacoustic/ultrasound dual imaging of human thyroid cancers: an initial clinical study," Biomed. Opt. Express 8(7), 3449-3457 (2017).

195. M. Toi et al., "Visualization of tumor-related blood vessels in human breast by photoacoustic imaging system with a hemispherical detector array," Sci. Rep. 7, 41970 (2017).

Paul Kumar Upputuri is currently a research fellow at Nanyang Technological University, Singapore. He has received his $\mathrm{PhD}$ in physics from Indian Institute of Technology (IIT) Madras in 2010. He has published around 80 papers in journals and conference proceedings. His research interests include optics, photoacoustics, biomedical imaging, molecular spectroscopy, super-resolution microscopy, multiphoton microscopy, and interferometry. $\mathrm{He}$ is a member of SPIE, OSA, Optics and Photonics Society of Singapore, Indian Laser Association, and Optical Society of India.

Manojit Pramanik is currently an assistant professor at the School of Chemical and Biomedical Engineering, Nanyang Technological University. He has received his $\mathrm{PhD}$ in biomedical engineering from Washington University in St. Louis, Missouri, USA. His research interests include the development of photoacoustic/thermoacoustic imaging systems, image reconstruction methods, clinical application areas, such as breast cancer imaging, molecular imaging, contrast agent development, and Monte Carlo simulation of light propagation in biological tissue. 